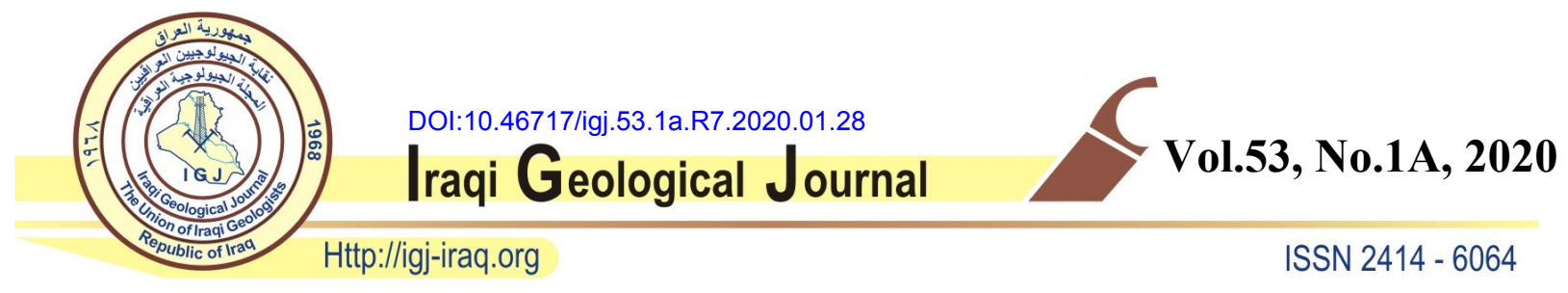

\title{
GEOCHEMICAL APPLICATION IN UNRAVELING PALEOWEATHERING, PROVENANCE AND ENVIRONMENTAL SETTING OF THE SHALE FROM CHIA GARA FORMATION, KURDISTAN REGION, IRAQ
}

\author{
${ }^{1}$ Rezhin K. Mustafa ${ }^{*}$ and $^{2}$ Faraj H. Tobia \\ ${ }^{1}$ College of Science, Salahaddin University, Erbil, Iraq \\ ${ }^{2}$ Department of Geology, College of Science, Salahaddin University, Erbil, Iraq \\ "E-mail: rezhin.mustafa@,su.edu.krd
}

Received: 17 March 2019; accepted: 13 July 2019

\begin{abstract}
The geochemical characteristics of the shale of the Chia Gara Formation (Middle TithonianBerriasian) from the Imbricated Zone (Barsarin section) and High Folded Zone (Banik section) Kurdistan Region, Iraq, was carried out to constrain their paleoweathering, provenance, and depositional environment. There are no clear differences in the major and trace elements of the Chia Gara Formation between the two studied sections. The chemical index of alteration (CIA) is significantly higher in the Barsarin than the Banik shales, suggesting more intense weathering of the Barsarin than the Banik shales. The samples of the Banik and some of Barsarin are clustered near the A-K line in A-CN-K plot suggests intense chemical weathering (high CIA) without any clear-cut evidence of K-metasomatism. The other samples of Barsarin have a weathering trend parallel to the A-CN line, indicating relatively steady state weathering conditions. The geochemical parameters of the shale $\left(\mathrm{Al}_{2} \mathrm{O}_{3} / \mathrm{TiO}_{2}\right.$, $T h / S c, L a / T h, L a / S c, L a / C o, T h / C o, C r / T h,(L a / L u)_{c n}$ and $\left.E u / E u{ }^{*}{ }_{c n}\right)$, and the diagrams ( $\mathrm{Th} / \mathrm{Sc}-\mathrm{Zr} / \mathrm{Sc}$ and $\mathrm{La} / \mathrm{Th}-\mathrm{Hf}$ ) indicate that they were derived from felsic (from the Rutba Uplift and/or Mosul High) and intermediate (from volcanic material during the spreading of Southern Neo-Tethys Ocean) components. The chondrite-normalized REE patterns are similar to those of Post Archean Australian Shale (PAAS), with the light rare earth element (REE) enrichment, a negative Eu anomaly, and almost flat heavy REE pattern. The geochemical parameters such as authigenic uranium, $\mathrm{U} / \mathrm{Th}, \mathrm{V} / \mathrm{Cr}, \mathrm{Ni} / \mathrm{Co}$, and $\mathrm{V} / \mathrm{Sc}$ ratios, and $\mathrm{Al}_{2} \mathrm{O}_{3}-\mathrm{V}$ and $\mathrm{Al}_{2} \mathrm{O}_{3}-\mathrm{P}_{2} \mathrm{O}_{5}$ diagrams indicate that these shales were deposited under deep marine suboxic to
\end{abstract}




\section{anoxic environment and shale of Banik section was deposited slightly under deeper environment than shale of Barsarin section.}

Keywords: Chia Gara Formation; Provenance; Paleoweathering; Shale; Geochemistry

\section{INTRODUCTION}

The geochemistry of clastic sedimentary rocks is used as an effective tool in the study of source area composition (Cullers, 2000, 2002; Osae et al., 2006; Armstrong-Altrin, 2009; Saha et al., 2010), intensity of chemical weathering in the source region (Selvaraj and Chen, 2006; Roy et al., 2008; Gallala et al., 2009; Gupta et al., 2012; Raza et al., 2012), paleoclimatic conditions (Nesbitt and Young, 1982; Bhatia, 1983; Taylor and McLennan, 1985; Absar et al., 2009; Raza et al., 2010), provenance (Cullers, 2000, 2002; Armstrong-Altrin, 2009; Bakkiaraj et al., 2010) and tectonic setting of the sedimentary basins (Cullers, 2000; Armstrong-Altrin and Verma, 2005; Joo et al., 2005; Sabaou et al., 2009; Fatima and Khan, 2012). The geochemical composition of clastic sediments is a complex function and depends on other factors such as physical sorting, diagenesis, source material and relief (Nagarajan et al., 2007a, 2007b; Armstrong-Altrin et al., 2004, 2012; Moosavirad et al., 2012). Likewise, geochemical parameters have been used by various authors to understand the paleo-oxygenation conditions of ancient sediments (Calvert and Pedersen, 1993; Jones and manning, 1994; Nath et al., 1997; Madhavaraju and Ramasamy, 1999; Cullers, 2002; Armstrong-Altrin et al., 2003; Dobrzinski et al., 2004). Determination of the provenance utilizing the elemental geochemistry in identifying source rocks for sedimentary rocks is based on the relative immobility of certain elements like La and Th that have high value in felsic rocks relative to basic rocks and $\mathrm{Co}, \mathrm{Cr}, \mathrm{Sc}$ and $\mathrm{Ni}$ have high values in basic rocks relative to felsic rocks within surficial environments in order that the elemental concentration or ratio reflects the composition of source rock much better than weathering and transportation. Therefore, types of rocks and environments are different with the difference in concentration and ratio of these elements (Roaldset, 1973; Cullers et al., 1975, 1979; Bavinton and Taylor, 1980; McLennan et al., 1983; Bhatia, 1985; Bhatia and Crook, 1986; Cullers, 1988; Wronkiewicz and Condie, 1990). The Chia Gara Formation is composed of organic matter-rich limestone and shale, it considered as an important petroleum source rocks (Odisho and Othman,1992; Al-Beyati,1998; Al-Ameri and Al-Obaidi, 2004). The Chia Gara Formation was introduced by Wetzel in 1950 (Bellen et al., 1959) in the Chia Gara anticline of the High Folded Zone. The type section of the formation comprises up to $230 \mathrm{~m}$ of thin bedded limestone and calcareous shale, with a consistent bullion zone and Phacoid beds at the base. Based on the fossil contents, the Chia Gara Formation 
indicates a Mid Tithonian-Berriasian age (Bellen et al., 1959; Jassim and Goff, 2006). The lithofacies of the Chia Gara Formation reflects the marine environment (Buday, 1980; Jassim and Goff, 2006) and Mohialdeen (2008) suggested the deep outer shelf to carbonate slope environments for the depositional setting. The present study examines the geochemistry of the shales of the Chia Gara Formation. The main purpose of this study is to determine the provenance, chemical weathering and the redox conditions for the shale of the Chia Gara Formation.

\section{GEOLOGICAL SETTING}

The Chia Gara Formation was studied throughout two sections: Barsarin and Banik (Fig. 1), belongs to the Late Tithonian- Hautrivian sedimentary sequence. It consists entirely of thin beds succession of limestone and shales (Fig. 2a\&2b) with rich ammonite faunas and diverse species of foraminifera, radiolarian, ostracodes and tintinnids. The lowermost $21 \mathrm{~m}$ is characterized by yellow marly limestones and shales (Bellen et al., 1959). The thickness of the formation ranges 30-300 m, $610 \mathrm{~m}$ of monotonous dark colored calcareous mudstones and rare marl bands were described in well Kirkuk-109 by McGinty in 1953 (Bellen et al., 1959) and referred to as the Karimia Mudstone. It conformably is underlain by the Barsarin Formation and conformablely overlain by the Lower Sarmord Formation (Fig. 2a\&2b). It was deposited during both transgression system tracks (TST) and high system track (HST) stages of the systems tract (Sharland et al., 2001).

Several authors studied the paleontology, stratigraphy and sedimentology of the Chia Gara Formation in Kurdistan Region (Howarth, 1992; Al-Qayim and Saadalla, 1992; Salae, 2001; Jassim and Goff, 2006; Mohialdeen, 2007; Mohialdeen and Al-Beyati, 2007; Mohialdeen, 2008). Sharland et al. (2001) suggest a regional unconformity at Mid Tithonian age on Arabian Plate which refers to the boundary between Megasequences AP7 (Late Toarcian- Early Tithonian) and AP8 (Late Tithonian- Early Turonian).

This may be a breakup unconformity which is a possible phase of ocean floor spreading during the opening of a Southern Neo- Tethys Ocean (Fig. 3). The opening of Southern NeoTethys led to the drifting away of a narrow microcontinent; a new passive margin formed along the NE margin of the Arabian Plate. The Rutba Uplift formed the western margin of Mesopotamian Basin. The NE margin was formed by a carbonate ridge along the north facing the passive margin of the Southern Neo-Tethys (Fig. 3). 


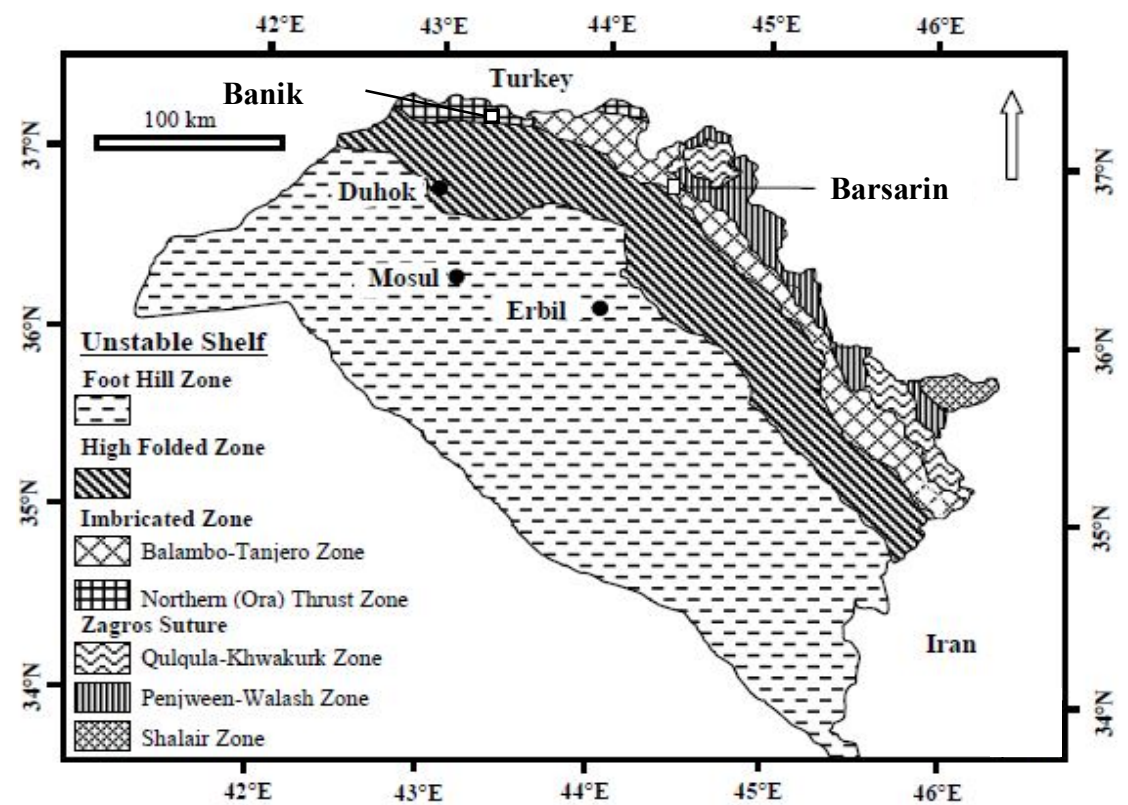

Fig. 1: Location map and tectonic zones of the Unstable Shelf, Iraq (modified from Buday and Jassim, 1984)
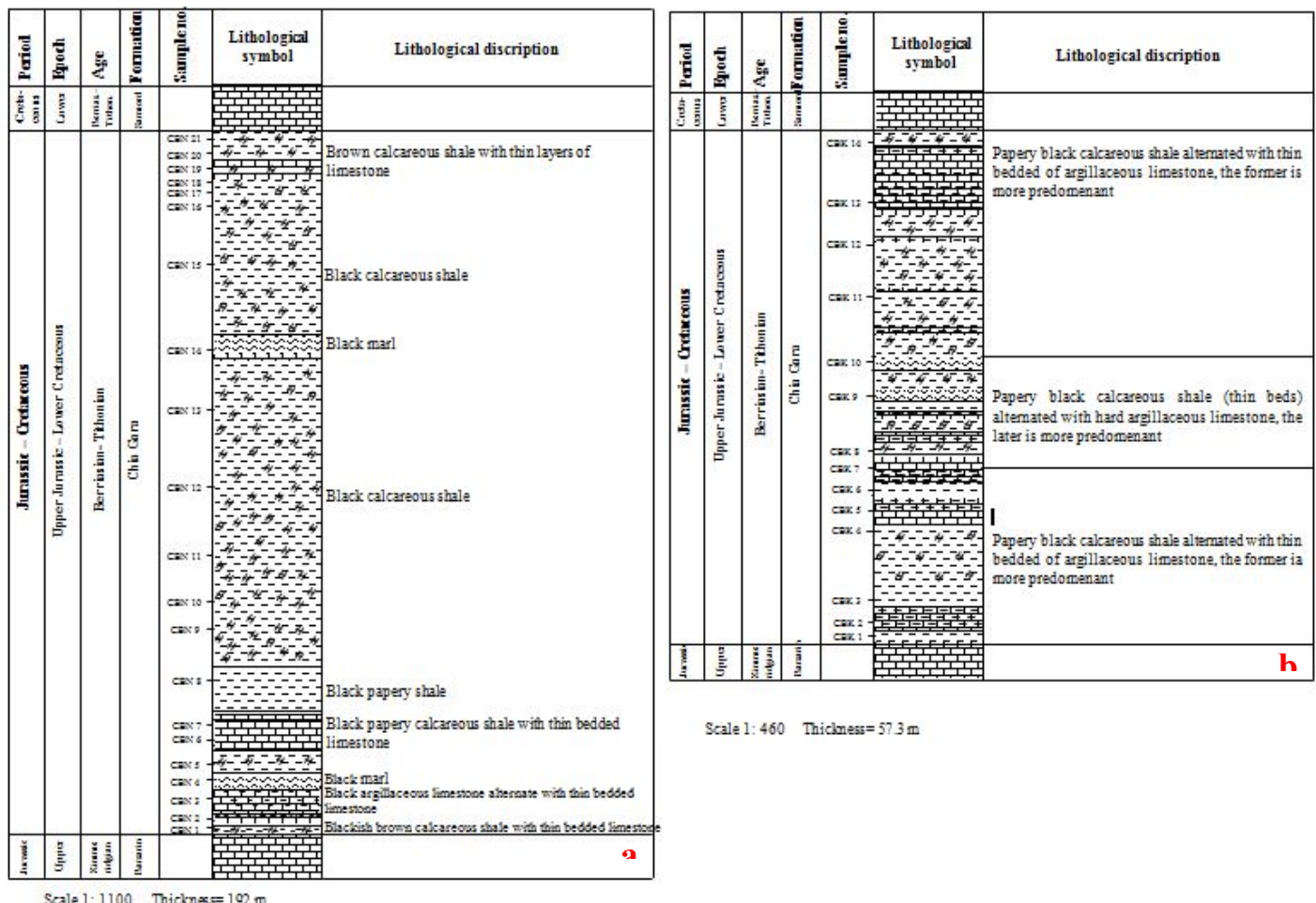

Scale 1: 460 Thickness $=573 \mathrm{~m}$

Fig. 2: Columnar sections for Chia Gara Formation, (a) Barsarin area (b) Banik area 


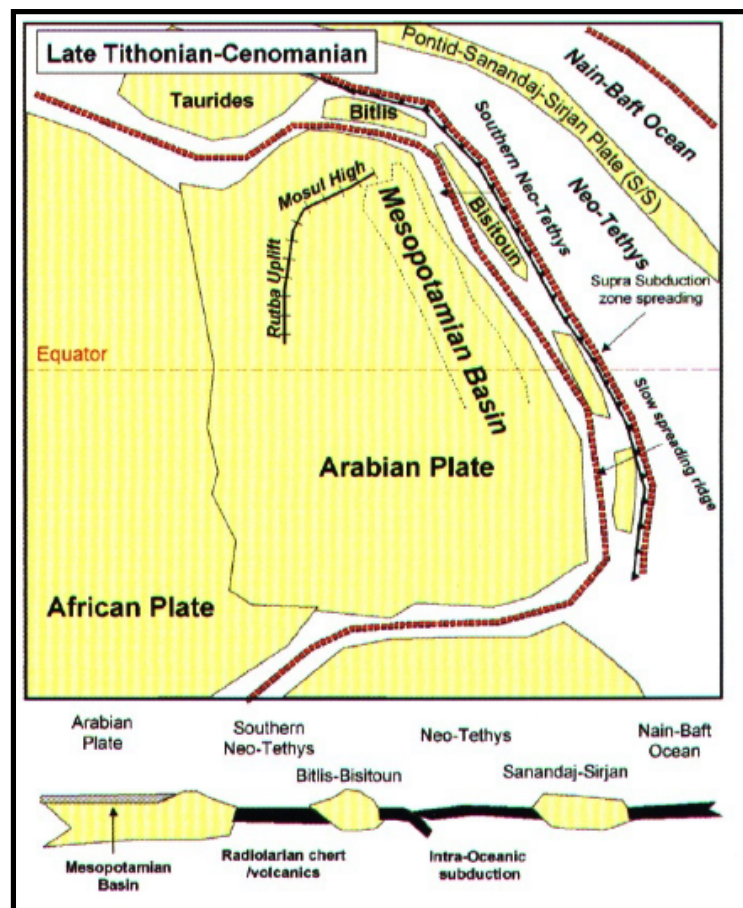

Fig. 3: Late Tithonian-Cenomanian geodynamic development of the Arabian Plate (Sharland et al., 2001)

\section{SAMPLING AND METHODS}

The samples were collected from two sections: the first section was near the Barsarin village at longitude 44 $39^{\prime} 13^{\prime \prime} \mathrm{N}$ and latitude $36^{\circ} 37^{\prime} 48^{\prime \prime} \mathrm{E}$ in the Imbricated Zone, and the second section was near Banik village at longitude 42 58'3.8' E and latitude $37^{\circ} 13^{\prime} 31.4^{\prime \prime} \mathrm{N}$ in the High Folded Zone (Fig. 1). Thirty five fresh samples were collected along these two sections; 21 samples from Barsarin and 14 samples from Banik (Fig. 2). The 35 samples were crushed, and then powdered to 200 mesh with an agate pulverizer. These samples were analyzed for major oxides $\left(\mathrm{SiO}_{2}\right.$, $\mathrm{Al}_{2} \mathrm{O}_{3}, \mathrm{Fe}_{2} \mathrm{O}_{3}, \mathrm{TiO}_{2}, \mathrm{CaO}, \mathrm{MgO}, \mathrm{MnO}, \mathrm{Na}_{2} \mathrm{O}, \mathrm{K}_{2} \mathrm{O}$ and $\mathrm{P}_{2} \mathrm{O}_{5}$ ) by inductively coupled plasmaatomic emission spectrometry (ICP-AES) after fused bead and acid digestion, under the analysis code ME-ICP06; trace elements (Rb, Sr, Ba, Th, U, Y, Zr, Nb, Hf, Sc, V, Cr, Co, Ni, Cu, Zn and Mo) and rare earth elements (La, Ce, Pr, Nd, Sm, Eu, Gd, Tb, Dy, Er, Ho, Tm, Yb, and Lu) were analyzed by inductively coupled plasma-mass spectrometry (ICP-MS) under the analysis code ME-MS81 at the ALS international laboratory in Spain. Loss on ignition (LOI) data was determined after drying the samples in oven $100{ }^{\circ} \mathrm{C}$ for $24 \mathrm{~h}$, to remove moisture content, next ignition the samples at $1000{ }^{\circ} \mathrm{C}$ for $2 \mathrm{~h}$. Chemical analysis of the major elements has precisions up to $5 \%$; whereas it varies between 0 and $9 \%$ for the trace and REEs. Internationally 
recognized standard materials OREAS-121 and AMIS0304 were used as references. Based on these standards, the accuracy and precision of the analysis were within $\pm 2 \%$ for elements $\mathrm{Nb}, \mathrm{Ni}$, $\mathrm{Zn}, \mathrm{Mo}, \mathrm{Ce}, \mathrm{Pr}, \mathrm{Sm}, \mathrm{Eu}, \mathrm{Tb}$ and Lu; \pm 5 \% for Sr, Ba, Th, Y, Zr, Co, Dy, Ho, Tm and Yb; and \pm 10 $\%$ for Rb, U, Hf, Sc, V, Cr, La, Nd, Gd and Er. Comparison of data between the present study and the published data of Post Archean Australian Shale (PAAS) and Upper Continental Crust (UCC) was done whenever possible. The REE data were normalized to the chondrite values of Taylor and McLennan (1985).

\section{RESULTS}

\section{Major Element Geochemistry}

The content of major oxides of the Chia Gara Formation is reported in Table 1. The results show few differences in composition among the two sections. In general, the shales of the Chia Gara Formation in Barsarin and Banik sections have high $\mathrm{CaO}$ content (11.55- 33.8, 22.28\% and 0.84$45.7,25.4 \%$, respectively). Such content has a great dilution effect on the other oxides, such as $\mathrm{SiO}_{2}$ content (23.2-46.5, 32.67\% and 9.01- 45.3, 27.66\%), $\mathrm{Al}_{2} \mathrm{O}_{3}(5.05-17.1,10.36 \%$ and 4.623.6, 11.19\%), $\mathrm{Fe}_{2} \mathrm{O}_{3}$ (1.8- 7.12, 4.04\% and 1.5- 8.65, 3.88\%), $\mathrm{MgO}(0.27-1.84,0.65 \%$ and 0.51$1.48,0.75 \%), \mathrm{Na}_{2} \mathrm{O}(0.02-0.58,0.16 \%$ and $0.01-0.52,0.10 \%), \mathrm{K}_{2} \mathrm{O}(0.5-1.65,0.90 \%$ and $0.45-$ 4.13, 2.53\%), $\mathrm{MnO}$ (0.01- 0.02, 0.011\% and 0.01- 0.03, 0.012\%), $\mathrm{TiO}_{2}(0.33-1.05,0.64 \%$ and $0.31-1.2,0.59 \%$ ). Except for $\mathrm{CaO}$, the studied shale shows depletion in all elements relative to those of the PAAS (Fig. 4). The $\mathrm{CaO}$ enrichment in these samples, as well as the significant correlation between $\mathrm{CaO}$ and LOI $(\mathrm{r}=0.903 ; \mathrm{n}=35)$, suggest that LOI and $\mathrm{CaO}$ are incorporated into calcite rather than other minerals. On the other hand, $\mathrm{Al}_{2} \mathrm{O}_{3}$ shows positive correlations with $\mathrm{SiO}_{2}, \mathrm{Fe}_{2} \mathrm{O}_{3}$, and $\mathrm{TiO}_{2}(\mathrm{r}=0.643,0.905$, and 0.947 , respectively; $\mathrm{n}=35)$.

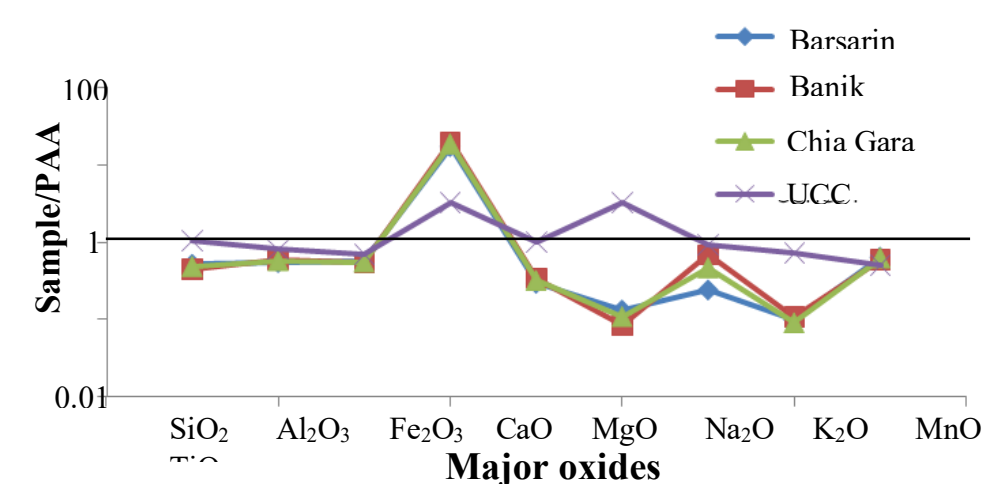

Fig. 4: UCC normalized spider diagrams for major oxides of the shale from Chia Gara Formation (PAAS values after Taylor and McLennan, 1985) 
Table 1: Major oxides contents (wt\%) for shale of the Chia Gara Formation; compared with PAAS (Taylor and McLennan, 1985) and UCC (Wedepohl, 1995)

\begin{tabular}{|c|c|c|c|c|c|c|c|c|c|c|c|c|}
\hline Sample no. & $\mathrm{SiO}_{2}$ & $\mathrm{Al}_{2} \mathrm{O}_{3}$ & $\mathrm{Fe}_{2} \mathrm{O}_{3}$ & $\mathrm{CaO}$ & $\mathrm{MgO}$ & $\mathrm{Na}_{2} \mathrm{O}$ & $\mathrm{K}_{2} \mathrm{O}$ & $\mathrm{MnO}$ & $\mathrm{TiO}_{2}$ & $\mathrm{P}_{2} \mathrm{O}_{5}$ & LOI & Total \\
\hline & \multicolumn{12}{|c|}{ Barsarin section } \\
\hline $\mathrm{C}$ & 25.4 & 12.25 & 6.48 & 24.2 & 0.81 & 0.04 & 1.65 & 0.02 & 0.66 & 0.54 & 26.3 & 98.35 \\
\hline CBN 2 & 27.1 & 9.79 & 3.23 & 27 & 0.72 & 0.11 & 1.1 & 0.01 & 0.57 & 0.24 & 28.9 & 98.77 \\
\hline CBN 3 & 23.9 & 7.06 & 2.55 & 33.6 & 0.67 & 0.23 & 1.05 & 0.01 & 0.4 & 0.13 & 30.0 & 99.60 \\
\hline CBN 4 & 25.6 & 5.05 & 1.8 & 33.8 & 0.78 & 0.38 & 0.7 & 0.01 & 0.33 & 0.09 & 29.5 & 98.04 \\
\hline CBN 5 & 36.0 & 6.47 & 2.9 & 25.4 & 0.49 & 0.58 & 0.82 & 0.01 & 0.4 & 0.03 & 25.0 & 98.10 \\
\hline CBN 6 & 27.6 & 7.62 & 3.61 & 27 & 0.7 & 0.5 & 0.9 & 0.01 & 0.47 & 0.05 & 24.3 & 92.76 \\
\hline CBN 7 & 32.1 & 6.64 & 3.58 & 19.15 & 0.29 & 0.51 & 0.77 & 0.01 & 0.43 & 0.06 & 19.4 & 82.94 \\
\hline CBN 8 & 46.5 & 13.4 & 6.39 & 12.8 & 0.72 & 0.35 & 1.51 & 0.02 & 0.85 & 0.08 & 17.75 & 100.37 \\
\hline CBN 9 & 42.1 & 12.4 & 3.86 & 15.05 & 0.83 & 0.15 & 1.35 & 0.01 & 0.89 & 0.09 & 18.4 & 95.13 \\
\hline CBN 10 & 23.2 & 5.78 & 2.2 & 31.9 & 1.84 & 0.02 & 0.52 & 0.01 & 0.36 & 0.01 & 30.0 & 95.84 \\
\hline CBN 11 & 32.3 & 8.68 & 3.23 & 23.9 & 0.94 & 0.02 & 0.86 & 0.01 & 0.59 & 0.07 & 23.1 & 93.70 \\
\hline CBN 12 & 33.4 & 9.26 & 3.52 & 23.6 & 0.66 & 0.05 & 0.98 & 0.01 & 0.59 & 0.1 & 22.4 & 94.57 \\
\hline CBN 13 & 44.7 & 11.1 & 4.07 & 14.3 & 0.73 & 0.03 & 1.08 & 0.01 & 0.73 & 0.09 & 17.75 & 94.59 \\
\hline CBN 14 & 33.9 & 8.15 & 2.94 & 26.5 & 0.41 & 0.04 & 0.81 & 0.01 & 0.56 & 0.05 & 22.9 & 96.27 \\
\hline CBN 15 & 33.8 & 10.65 & 4.37 & 20.7 & 0.37 & 0.04 & 0.66 & 0.01 & 0.67 & 0.09 & 21.2 & 92.56 \\
\hline CBN 16 & 34.9 & 16.55 & 6.54 & 11.55 & 0.53 & 0.08 & 1.06 & 0.01 & 1.05 & 0.14 & 20.1 & 92.51 \\
\hline CBN 17 & 33.6 & 14.35 & 4.63 & 15.55 & 0.77 & 0.07 & 0.7 & 0.01 & 0.84 & 0.08 & 19.3 & 89.90 \\
\hline CBN 18 & 33.7 & 11.6 & 3.88 & 20.5 & 0.3 & 0.04 & 0.57 & 0.01 & 0.69 & 0.09 & 21.2 & 92.58 \\
\hline CBN 19 & 4 & 12.25 & 3 & 17 & 7 & 0.02 & 0.55 & 0.01 & 0.71 & 13 & 19.7 & 93.82 \\
\hline CBN 20 & 26.1 & 11.35 & 3.83 & 24.7 & 0.29 & 0.02 & 0.5 & 0.01 & 0.7 & 0.08 & 23.9 & 91.48 \\
\hline CBN 21 & 31.7 & 17.1 & 7.12 & 18.85 & 0.44 & 0.1 & 0.86 & 0.01 & 0.92 & 0.21 & 22.8 & 100.11 \\
\hline \multirow[t]{2}{*}{ Average } & 2.67 & 10.36 & 4.04 & 22.28 & 0.65 & 0.16 & 0.90 & 0.011 & 0.64 & 0.12 & 23.04 & 94.87 \\
\hline & \multicolumn{12}{|c|}{ Banik section } \\
\hline CBK 1 & 45.3 & 23.3 & 8.65 & 0.84 & 1.48 & 0.05 & 3.5 & 0.03 & 1.13 & 0.2 & 16.95 & 101.43 \\
\hline CBK 2 & 9.01 & 4.6 & 1.5 & 45.7 & 0.62 & 0.01 & 0.62 & 0.01 & 0.24 & 0.06 & 38.8 & 101.17 \\
\hline CBK 3 & 42.9 & 23.6 & 7.64 & 1.81 & 1.06 & 0.05 & 2.67 & 0.02 & 1.2 & 0.24 & 18.85 & 100.04 \\
\hline CBK 4 & 27.8 & 10.85 & 3.45 & 23.3 & 0.89 & 0.05 & 2.95 & 0.01 & 0.48 & 0.28 & 28.6 & 98.66 \\
\hline CBK 5 & 24.2 & 8.26 & 2.45 & 31.2 & 0.73 & 0.08 & 2.42 & 0.01 & 0.41 & 0.25 & 30.9 & 100.91 \\
\hline CBK 6 & 32.9 & 11.1 & 2.85 & 21.1 & 0.77 & 0.14 & 4.13 & 0.01 & 0.47 & 0.23 & 25.6 & 99.30 \\
\hline CBK 7 & 30.6 & 8.09 & 2.95 & 27.1 & 0.62 & 0.24 & 3.87 & 0.01 & 0.38 & 0.11 & 26.3 & 100.27 \\
\hline CBK 8 & 32.6 & 9.29 & 3.72 & 22.1 & 0.65 & 0.52 & 3.97 & 0.01 & 0.51 & 0.22 & 25.5 & 99.09 \\
\hline CBK 9 & 20.7 & 6.51 & 2.71 & 34.3 & 0.71 & 0.04 & 2.48 & 0.01 & 0.37 & 0.12 & 31.1 & 99.05 \\
\hline CBK 10 & 26.9 & 9.28 & 2.94 & 28.1 & 0.62 & 0.05 & 3.01 & 0.01 & 0.54 & 0.17 & 28.3 & 99.92 \\
\hline CBK 11 & 27.8 & 9.69 & 4.51 & 27.2 & 0.61 & 0.04 & 2.86 & 0.01 & 0.57 & 0.11 & 26.9 & 100.3 \\
\hline CBK 12 & 35.3 & 15.35 & 6.66 & 14.75 & 0.65 & 0.01 & 1.71 & 0.01 & 0.93 & 0.4 & 22.6 & 98.37 \\
\hline CBK 13 & 10.4 & 4.82 & 1.63 & 45.7 & 0.51 & 0.02 & 0.45 & 0.01 & 0.31 & 0.28 & 37.3 & 101.43 \\
\hline CBK 14 & 20.8 & 11.9 & 2.69 & 32.4 & 0.58 & 0.03 & 0.75 & 0.01 & 0.76 & 0.08 & 30.7 & 100.70 \\
\hline Average & 27.66 & 11.19 & 3.889 & 25.4 & 0.75 & 0.10 & 2.53 & 0.012 & 0.59 & 0.20 & 27.74 & 100.06 \\
\hline Total avg. & 30.17 & 10.78 & 3.96 & 23.84 & 0.70 & 0.13 & 1.72 & 0.01 & 0.62 & 0.16 & 25.39 & 97.48 \\
\hline PAAS & 62.40 & 18.78 & 7.18 & 1.29 & 2.19 & 1.19 & 3.68 & 0.11 & 0.99 & 0.16 & 6.00 & \\
\hline $\mathrm{UCC}$ & 66.00 & 15.20 & 5.00 & 4.20 & 2.20 & 3.90 & 3.40 & 0.08 & 0.50 & - & - & \\
\hline
\end{tabular}




\section{Trace Element Geochemistry}

The trace element content of the Chia Gara Formation is given in (Table 2). The studied shale shows enrichment of $\mathrm{Sr}, \mathrm{U}, \mathrm{V}, \mathrm{Ni}$ and depletion in $\mathrm{Rb}, \mathrm{Ba}, \mathrm{Th}, \mathrm{Y}, \mathrm{Zr}, \mathrm{Nb}, \mathrm{Hf}, \mathrm{Sc}$, and Co relative to PAAS (Fig. 5). The enrichment of the shale with Sr at Barsarin section (112- 877, 769 ppm) indicates the association with the carbonate phase, especially calcite mineral, which is higher than that of the Banik section $(132-403,260 \mathrm{ppm})$. On the other hand, the $\mathrm{Al}_{2} \mathrm{O}_{3}$ content was significantly correlated with $\mathrm{Rb}, \mathrm{Th}, \mathrm{Y}, \mathrm{Zr}, \mathrm{Nb}, \mathrm{Hf}, \mathrm{Sc}$, and REE, indicate their association with the detrital phase.

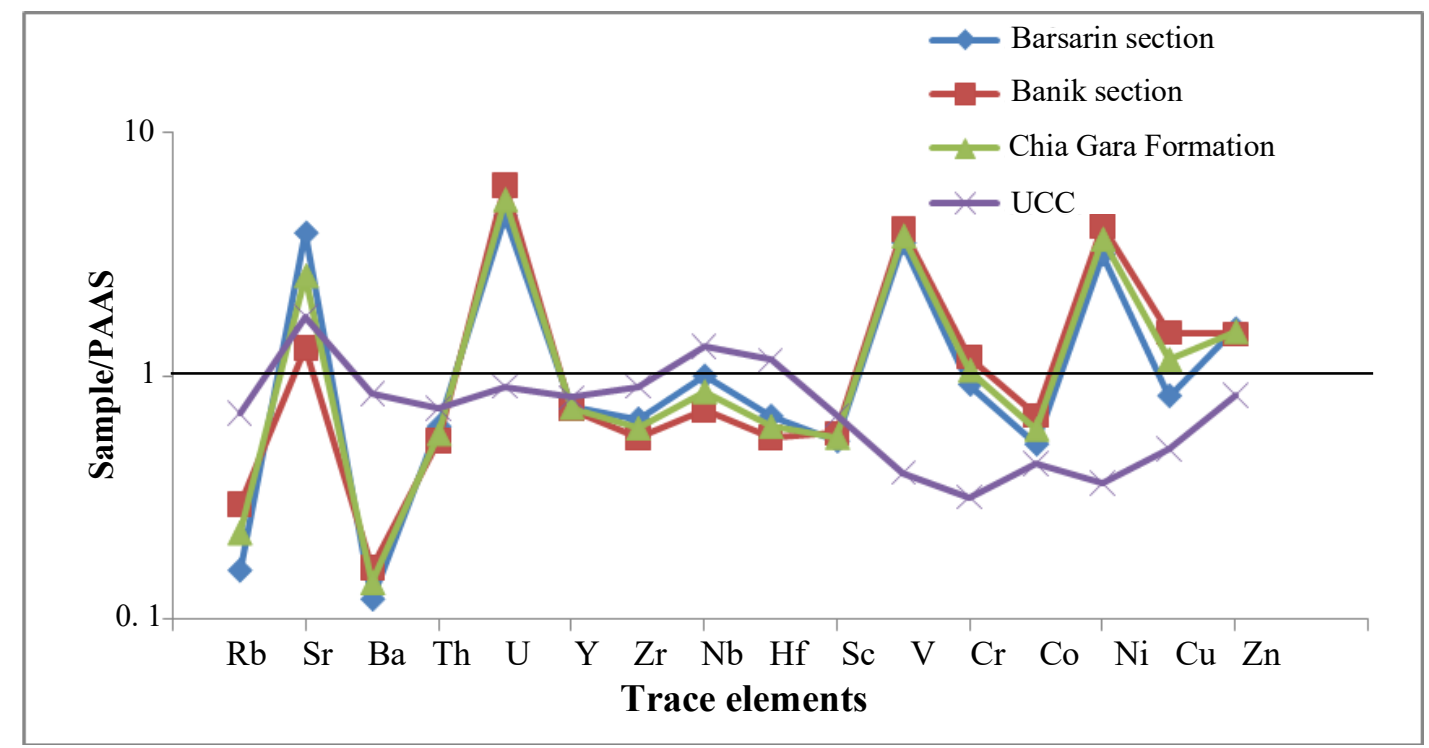

Fig. 5: PAAS normalized spider diagrams for trace elements of the shale from Chia Gara Formation (PAAS values after Taylor and McLennan, 1985)

\section{Rare Earth Element Geochemistry}

The content of total rare earth elements ( $\mathrm{REE})$ in the Chia Gara shale varies from $39.78 \mathrm{ppm}$ to $196.41 \mathrm{ppm}$ with an average of $123.25 \mathrm{ppm}$, significantly lower than for upper continental crust (UCC; $146.37 \mathrm{ppm}$ ) and PAAS (184.77 ppm; Table 3). It is suggested that the effect of dilution by carbonate materials, is the major control on the REE contents (significant correlation between $\Sigma \mathrm{REE}$ and $\mathrm{CaO}$ is -0.854). $\Sigma \mathrm{REE}$ have a significant correlation with $\mathrm{SiO}_{2}, \mathrm{Al}_{2} \mathrm{O}_{3}, \mathrm{Fe}_{2} \mathrm{O}_{3}$, and $\mathrm{TiO}_{2}(0.751,0.869,0.858$, and 0.937 , respectively) suggest the typical role of clay minerals on the distribution of REEs (McLennan, 1989; Condie, 1991). 
Table 2: Trace element concentrations (ppm) for shale of the Chia Gara Formation; compared with PAAS (Taylor and McLennan, 1985) and UCC (Wedepohl, 1995)

\begin{tabular}{|c|c|c|c|c|c|c|c|c|c|c|c|c|c|c|c|c|c|}
\hline & \multicolumn{3}{|c|}{ Large ion lithophile elements } & \multicolumn{6}{|c|}{ High field strength elements } & \multicolumn{8}{|c|}{ Transition elements } \\
\hline & $\mathrm{Rb}$ & $\mathrm{Sr}$ & $\mathrm{Ba}$ & Th & $\mathrm{U}$ & $\mathrm{Y}$ & $\mathrm{Zr}$ & $\mathrm{Nb}$ & $\mathrm{Hf}$ & $\mathrm{Sc}$ & V & $\mathrm{Cr}$ & Co & $\mathrm{Ni}$ & $\mathrm{Cu}$ & $\mathrm{Zn}$ & Mo \\
\hline & \multicolumn{17}{|c|}{ Barsarin section } \\
\hline CBN 1 & 57.5 & 1085 & 91.9 & 8.86 & 51.4 & 15.7 & 132 & 18 & 3 & 11 & 619 & 90 & 65 & 283 & 141 & 184 & 502 \\
\hline CBN 2 & 31.7 & 1505 & 367 & 7.31 & 22.8 & 20.9 & 110 & 15.4 & 2.8 & 7 & 684 & 90 & 15 & 212 & 51 & 117 & 351 \\
\hline CBN 3 & 25.5 & 1120 & 72.9 & 5.04 & 18.7 & 21.8 & 86 & 10.7 & 2 & 6 & 908 & 100 & 7 & 234 & 55 & 156 & 288 \\
\hline CBN 4 & 14.9 & 1420 & 50 & 3.94 & 17.25 & 18.6 & 70 & 9 & 1.6 & 5 & 600 & 110 & 5 & 217 & 34 & 131 & 171 \\
\hline CBN 5 & 16.4 & 747 & 54.9 & 5.36 & 15 & 18.2 & 81 & 10.6 & 1.8 & 6 & 287 & 70 & 16 & 166 & 41 & 116 & 155 \\
\hline CBN 6 & 18 & 877 & 41.4 & 6.69 & 16.35 & 19 & 100 & 13.6 & 2.3 & 8 & 295 & 80 & 9 & 140 & 26 & 111 & 104 \\
\hline CBN 7 & 14.5 & 531 & 50.7 & 6.43 & 9.59 & 14.2 & 91 & 12.6 & 2 & 6 & 462 & 80 & 8 & 241 & 34 & 162 & 114 \\
\hline CBN 8 & 34.2 & 817 & 94.1 & 12.1 & 10.85 & 32.1 & 169 & 23.4 & 4.6 & 13 & 564 & 150 & 15 & 224 & 40 & 177 & 128 \\
\hline CBN 9 & 31.2 & 785 & 70.1 & 10.6 & 9.93 & 25.8 & 203 & 25.5 & 5.2 & 11 & 725 & 140 & 9 & 180 & 41 & 201 & 96 \\
\hline CBN 10 & 13.2 & 1165 & 36 & 5.26 & 11.3 & 16.4 & 92 & 11.6 & 2 & 5 & 287 & 60 & 6 & 81 & 17 & 63 & 63 \\
\hline CBN 11 & 21 & 831 & 45.2 & 7.7 & 13 & 22.0 & 131 & 17.9 & 3.3 & 8 & 482 & 80 & 6 & 163 & 30 & 110 & 146 \\
\hline CBN 12 & 26.4 & 725 & $\begin{array}{l}63.8 \\
\end{array}$ & 7.54 & 14.4 & 18.8 & 139 & 17.9 & 3.6 & 8 & 440 & 90 & 8 & 157 & 30 & 99 & 120 \\
\hline CBN 13 & 28.3 & 573 & 54.6 & 9.8 & 11.65 & 21.8 & 163 & 21.9 & 3.7 & 10 & 639 & 110 & 8 & 226 & 41 & 154 & 157 \\
\hline CBN 14 & 20.8 & 868 & 47 & 7.39 & 7.23 & 17.6 & 125 & 16.4 & 3 & 7 & 482 & 80 & 7 & 100 & 30 & 122 & 56 \\
\hline CBN 15 & 21.7 & 706 & 65.6 & 9.96 & 14.55 & 22.4 & 153 & 20.2 & 3.7 & 10 & 572 & 90 & 9 & 210 & 40 & 114 & 208 \\
\hline CBN 16 & 34.4 & 465 & 73.3 & 15.25 & 7.76 & 23.9 & 235 & 31.8 & 5.6 & 12 & 677 & 140 & 14 & 189 & 40 & 168 & 111 \\
\hline CBN 17 & 24.9 & 379 & 75.9 & 13.4 & 7.44 & 18 & 183 & 26 & 4.9 & 10 & 500 & 100 & 10 & 116 & 31 & 112 & 91 \\
\hline CBN 18 & 21.4 & 412 & 65.3 & 11.8 & 10.8 & 18.6 & 166 & 23.8 & 4.3 & 9 & 447 & 90 & 7 & 138 & 40 & 107 & 126 \\
\hline CBN 19 & 20.7 & 378 & 69 & 11.4 & 10.3 & 18.5 & 161 & 22.6 & 4.4 & 9 & 368 & 100 & 8 & 139 & 36 & 100 & 172 \\
\hline CBN 20 & 19.2 & 402 & 63.5 & 9.5 & 6.36 & 16.9 & 133 & 19.6 & 3.4 & 9 & 377 & 80 & 9 & 105 & 24 & 98 & 68 \\
\hline CBN 21 & 36.3 & 363 & 91.1 & 14.15 & 7.98 & 23.5 & 195 & 27.6 & 5 & 10 & 604 & 190 & 10 & 108 & 43 & 160 & 58 \\
\hline \multirow[t]{2}{*}{ Average } & 25.3 & 769 & 78.3 & 9.0 & 14.0 & 20.2 & 139 & 18.9 & 3.4 & 8.6 & 525 & 101 & 12.0 & 173 & 41.2 & 132 & 156.4 \\
\hline & \multicolumn{17}{|c|}{ Banik section } \\
\hline CBK 1 & 105.5 & 132 & 198 & 16.3 & 20.5 & 21.4 & 200 & 27 & 5.2 & 18 & 686 & 150 & 46 & 197 & 95 & 144 & 206 \\
\hline CBK 2 & 18 & 138 & 44.1 & 2.86 & 7.99 & 5.5 & 45 & 5.8 & 1 & $T$ & 124 & 30 & 8 & 47 & 15 & 24 & 37 \\
\hline CBK 3 & 82 & 156 & 169 & 16.3 & 20.7 & 24.2 & 214 & 29.2 & 5.4 & 18 & 442 & 150 & 33 & 189 & 75 & 131 & 220 \\
\hline CBK 4 & 58.4 & 232 & 126 & 7.04 & 31.9 & 16.3 & 91 & 9.8 & 2 & 10 & 697 & 90 & 15 & 212 & 102 & 135 & 333 \\
\hline CBK 5 & 45.7 & 403 & 121 & 5.24 & 29.6 & 12.9 & 97 & 8.1 & 2.1 & 7 & 651 & 100 & 10 & 168 & 58 & 84 & 314 \\
\hline CBK 6 & 63.2 & 189 & 150.5 & 7.06 & 25.3 & 18 & 89 & 9.6 & 2.1 & 9 & 726 & 110 & 14 & 233 & 99 & 140 & 242 \\
\hline CBK 7 & 38.1 & 270 & 73.8 & 5.89 & 14.75 & 15.4 & 71 & 9.2 & 1.6 & 6 & 428 & 110 & 10 & 243 & 72 & 120 & 134 \\
\hline CBK 8 & 44.1 & 268 & 127.5 & 6.97 & 22.2 & 26 & 117 & 13 & 2.7 & 8 & 1020 & 170 & 13 & 400 & 124 & 147 & 266 \\
\hline CBK 9 & 34.5 & 306 & 61.2 & 4.88 & 14.9 & 15.8 & 74 & 9 & 1.7 & 6 & 521 & 100 & 5 & 199 & 69 & 104 & 132 \\
\hline CBK 10 & 40.6 & 268 & 88.7 & 6.79 & 17.8 & 22.2 & 114 & 12.3 & 2.7 & 8 & 522 & 120 & 11 & 269 & 71 & 126 & 121 \\
\hline CBK 11 & 38.7 & 272 & 85.5 & 7.64 & 20.4 & 24.5 & 117 & 13.8 & 2.8 & 8 & 652 & 140 & 24 & 384 & 115 & 191 & 154 \\
\hline CBK 12 & 47.2 & 227 & 121 & 11.95 & 17.05 & 32.6 & 177 & 20.7 & 4.1 & 13 & 1100 & 210 & 19 & 393 & 84 & 150 & 278 \\
\hline CBK 13 & 16.4 & 392 & 34.5 & 3.77 & 8.69 & 17.5 & 70 & 7.4 & 1.4 & 5 & 430 & 80 & 6 & 129 & 41 & 199 & 47 \\
\hline CBK 14 & 30.4 & 392 & 79.9 & 8.4 & 10.45 & 23.5 & 162 & 18.5 & 4 & 10 & 381 & 280 & 7 & 118 & 31 & 68 & 23 \\
\hline Average & 47.3 & 260 & 105.7643 & 7.9 & 18.7 & 19.7 & 117 & 13.8 & 2.8 & 9.3 & 599 & 131 & 15.8 & 227 & 75.1 & 126 & 179 \\
\hline Total av & 36.3 & 514 & 92.0 & 8.45 & 16.35 & 20.0 & 128 & 16.35 & 3.1 & 8.9 & 562 & 116 & 13.9 & 200 & 58.2 & 129 & 168 \\
\hline PAAS & 160 & 200 & 650 & 14.6 & 3.1 & 27 & 210 & 19 & 5 & 16 & 150 & 110 & 23 & 55 & 50 & 85 & - \\
\hline UCC & 112 & 350 & 550 & 10.7 & 2.8 & 22 & 190 & 25 & 5.8 & 11 & 60 & 35 & 10 & 20 & 25 & 71 & 1.5 \\
\hline
\end{tabular}


The chondrite normalized REE patterns (Taylor and McLennan, 1985) of the studied shales are given in Figure (6). The moderate negative $\mathrm{Eu}$ anomalies $\left(\mathrm{Eu} / \mathrm{Eu}^{*} \mathrm{cn}\right.$ is between 0.59 and 0.75 with an average of 0.67 ) which are attributed to the Eu-depletion in the source area. The Chia Gara samples have highly enriched light rare earth element (LREE) patterns where $(\mathrm{La} / \mathrm{Sm})_{\mathrm{cn}}=4.02$; moreover, these are slightly lower than UCC (4.20) and PAAS (4.33; Taylor and McLennan, 1985). The slightly higher $(\mathrm{La} / \mathrm{Yb})_{\mathrm{cn}}$ ratios $(7.94-12.07,9.79$; Table 4) than UCC (9.21) and PAAS (9.15), indicate the LREE enrichment. The patterns show enrichment in light REE (LREE) and depletion in heavy REE (HREE) in addition to the flat HREE pattern. The Chia Gara shale has flat to moderately fractionated HREE patterns which $(\mathrm{Gd} / \mathrm{Yb})_{\mathrm{cn}}=1.47$ (Table 4).

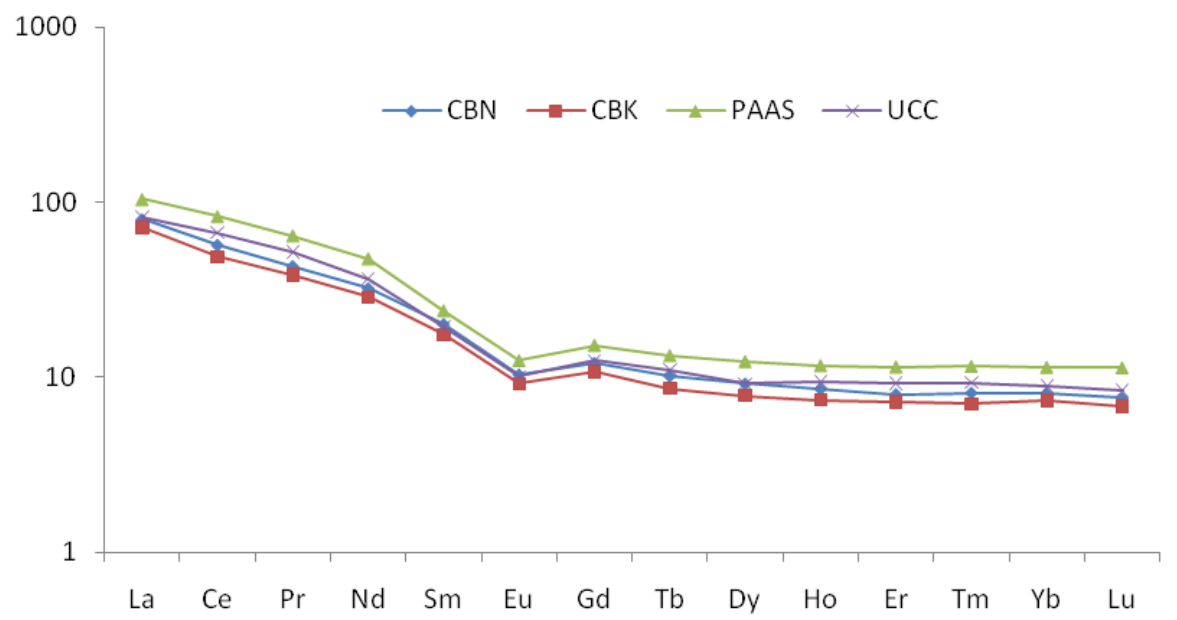

Fig. 6: Chondrite normalized rare earth elements, the plot for shale samples from Chia Gara (Taylor and McLennan, 1985) 
Table 3: Rare earth element concentrations (ppm) for shale of the Chia Gara Formation

\begin{tabular}{|c|c|c|c|c|c|c|c|c|c|c|c|c|c|c|c|}
\hline Sample no. & $\mathrm{La}$ & $\mathrm{Ce}$ & $\operatorname{Pr}$ & $\mathrm{Nd}$ & $\mathrm{Sm}$ & $\mathrm{Eu}$ & Gd & $\mathrm{Tb}$ & Dy & Ho & $\mathrm{Er}$ & $\mathrm{Tm}$ & $\mathrm{Yb}$ & $\mathrm{Lu}$ & $\Sigma$ REE \\
\hline CBN 1 & 24.8 & 52.1 & 5.22 & 20.4 & 4.32 & 0.85 & 3.24 & 0.52 & 2.98 & 0.63 & 1.63 & 0.21 & 1.59 & 0.24 & 118.73 \\
\hline CBN 2 & 28.5 & 48.4 & 5.47 & 21.7 & 4.26 & 0.84 & 3.38 & 0.51 & 3.16 & 0.7 & 1.78 & 0.27 & 1.71 & 0.27 & 120.95 \\
\hline CBN 3 & 21.7 & 34.6 & 4.28 & 17.2 & 3.73 & 0.69 & 3.12 & 0.48 & 3.03 & 0.62 & 1.75 & 0.26 & 1.84 & 0.22 & 93.52 \\
\hline CBN 4 & 19.1 & 29.1 & 3.68 & 14.5 & 2.97 & 0.61 & 2.67 & 0.41 & 2.57 & 0.57 & 1.55 & 0.22 & 1.44 & 0.19 & 79.58 \\
\hline CBN 5 & 21.6 & 41.2 & 4.67 & 19 & 3.8 & 0.83 & 3.07 & 0.51 & 3.02 & 0.65 & 1.49 & 0.24 & 1.61 & 0.23 & 101.92 \\
\hline CBN 6 & 23.9 & 49.6 & 5.56 & 21.4 & 4.74 & 0.84 & 3.7 & 0.58 & 3.28 & 0.7 & 1.91 & 0.27 & 1.71 & 0.23 & 118.42 \\
\hline CBN 7 & 21 & 39.9 & 4.35 & 17.5 & 3.4 & 0.66 & 2.69 & 0.39 & 2.45 & 0.51 & 1.48 & 0.2 & 1.5 & 0.2 & 96.23 \\
\hline CBN 8 & 41 & 81.2 & 8.65 & 33.9 & 7.58 & 1.48 & 6.25 & 0.98 & 5.49 & 1.14 & 2.98 & 0.45 & 2.91 & 0.42 & 194.43 \\
\hline CBN 9 & 33 & 61.1 & 6.62 & 26 & 5.12 & 0.98 & 4.01 & 0.73 & 4.5 & 0.92 & 2.5 & 0.39 & 2.64 & 0.4 & 148.91 \\
\hline CBN 10 & 18.8 & 37.9 & 4.14 & 16.9 & 3.41 & 0.78 & 3.21 & 0.47 & 2.68 & 0.57 & 1.63 & 0.2 & 1.6 & 0.23 & 92.52 \\
\hline CBN 11 & 25.6 & 47.3 & 5.02 & 19.9 & 4.53 & 0.91 & 3.56 & 0.58 & 3.62 & 0.72 & 2.09 & 0.3 & 2.05 & 0.32 & 116.5 \\
\hline CBN 12 & 25.7 & 46.5 & 5.09 & 19.6 & 3.81 & 0.69 & 3.37 & 0.55 & 3.02 & 0.67 & 1.89 & 0.28 & 1.83 & 0.28 & 113.28 \\
\hline CBN 13 & 30.1 & 55.2 & 6.02 & 24 & 4.85 & 0.96 & 3.54 & 0.61 & 3.73 & 0.78 & 2.12 & 0.34 & 2.18 & 0.32 & 134.75 \\
\hline $\mathrm{CBN} 14$ & 25.6 & 47.8 & 5.23 & 20.3 & 4.08 & 0.87 & 3.56 & 0.54 & 3.1 & 0.66 & 1.89 & 0.25 & 1.9 & 0.24 & 116.02 \\
\hline CBN 15 & 31.3 & 60.1 & 6.72 & 25.5 & 5.2 & 1.04 & 4.03 & 0.7 & 4.03 & 0.84 & 2.23 & 0.35 & 2.17 & 0.3 & 144.51 \\
\hline CBN 16 & 42.1 & 76.2 & 7.91 & 29.8 & 5.76 & 1.07 & 4.29 & 0.73 & 4.3 & 0.89 & 2.57 & 0.38 & 2.75 & 0.41 & 179.16 \\
\hline $\mathrm{CBN} 17$ & 39.1 & 70.9 & 7.26 & 26.7 & 4.98 & 0.91 & 3.6 & 0.57 & 3.38 & 0.76 & 2.05 & 0.32 & 2.31 & 0.3 & 163.14 \\
\hline CBN 18 & 35 & 66.3 & 6.78 & 25.6 & 5.14 & 0.87 & 3.84 & 0.6 & 3.68 & 0.72 & 2.05 & 0.31 & 1.96 & 0.32 & 153.17 \\
\hline CBN 19 & 35.5 & 67.3 & 7.18 & 27 & 5.54 & 0.99 & 4.05 & 0.59 & 3.73 & 0.74 & 1.96 & 0.3 & 2.04 & 0.32 & 157.24 \\
\hline CBN 20 & 29.9 & 55.5 & 6.07 & 23.4 & 4.66 & 0.87 & 3.67 & 0.61 & 3.45 & 0.71 & 1.84 & 0.24 & 1.85 & 0.27 & 133.04 \\
\hline CBN 21 & 42.8 & 79.4 & 8.36 & 31.3 & 5.87 & 1.16 & 4.58 & 0.74 & 4.38 & 0.91 & 2.45 & 0.4 & 2.58 & 0.35 & 185.28 \\
\hline Average & 29.34 & 54.652 & 5.92 & 22.93 & 4.65 & 0.9 & 3.69 & 0.59 & 3.5 & 0.73 & 1.99 & 0.29 & 2.01 & 0.29 & 131.482 \\
\hline CBK 1 & 40 & 77.5 & 7.82 & 29.6 & 6.04 & 1.04 & 4.11 & 0.64 & 4.18 & 0.78 & 2.22 & 0.36 & 2.41 & 0.36 & 177.06 \\
\hline CBK 2 & 9 & 17.4 & 1.69 & 6.7 & 1.18 & 0.24 & 1.02 & 0.14 & 0.89 & 0.19 & 0.6 & 0.07 & 0.6 & 0.06 & 39.78 \\
\hline CBK 3 & 43.7 & 84.5 & 8.88 & 33.4 & 6.93 & 1.25 & 5.22 & 0.77 & 4.68 & 0.95 & 2.43 & 0.41 & 2.85 & 0.44 & 196.41 \\
\hline CBK 4 & 22.9 & 42.7 & 4.78 & 18.4 & 3.54 & 0.79 & 3.13 & 0.47 & 2.79 & 0.55 & 1.58 & 0.21 & 1.57 & 0.22 & 103.63 \\
\hline CBK 5 & 18.9 & 31.8 & 3.72 & 14 & 2.55 & 0.58 & 2.15 & 0.32 & 1.97 & 0.39 & 1.09 & 0.15 & 1.14 & 0.17 & 78.93 \\
\hline CBK 6 & 25.7 & 45 & 5.18 & 20.4 & 3.77 & 0.83 & 3.25 & 0.51 & 2.68 & 0.57 & 1.57 & 0.23 & 1.61 & 0.2 & 111.5 \\
\hline CBK 7 & 20.3 & 34.5 & 3.88 & 15.1 & 3.24 & 0.69 & 2.47 & 0.37 & 2.1 & 0.47 & 1.23 & 0.16 & 1.3 & 0.17 & 85.98 \\
\hline CBK 8 & 25.1 & 41.7 & 5.01 & 19.9 & 4.24 & 0.8 & 3.53 & 0.53 & 3.19 & 0.77 & 2.29 & 0.31 & 1.96 & 0.32 & 109.65 \\
\hline CBK 9 & 18.2 & 29.2 & 3.45 & 13.7 & 2.6 & 0.46 & 2.12 & 0.35 & 2.05 & 0.43 & 1.27 & 0.18 & 1.28 & 0.19 & 75.48 \\
\hline CBK 10 & 26.2 & 45 & 5.35 & 21.2 & 3.93 & 0.8 & 3.33 & 0.53 & 3.13 & 0.67 & 1.98 & 0.27 & 1.93 & 0.24 & 114.56 \\
\hline CBK 11 & 30.1 & 50.9 & 5.96 & 24 & 4.33 & 0.88 & 3.93 & 0.58 & 3.62 & 0.78 & 2.12 & 0.32 & 2.08 & 0.32 & 129.92 \\
\hline CBK 12 & 40 & 75.2 & 8.32 & 31.8 & 6.62 & 1.3 & 5.69 & 0.84 & 4.85 & 1.06 & 2.93 & 0.4 & 3.04 & 0.43 & 182.48 \\
\hline CBK 13 & 17.1 & 28.9 & 3.61 & 14.3 & 2.95 & 0.61 & 2.41 & 0.37 & 2.23 & 0.49 & 1.38 & 0.15 & 1.33 & 0.19 & 76.02 \\
\hline CBK 14 & 30.6 & 48.9 & 5.94 & 22.8 & 4.73 & 0.94 & 3.58 & 0.59 & 3.43 & 0.76 & 2.33 & 0.33 & 2.41 & 0.34 & 127.68 \\
\hline Average & 26.27 & 46.66 & 5.26 & 20.38 & 4.05 & 0.80 & 3.28 & 0.50 & 2.99 & 0.63 & 1.79 & 0.25 & 1.82 & 0.26 & 114.94 \\
\hline Total av & 27.81 & 50.66 & 5.59 & 21.66 & 4.35 & 0.85 & 3.49 & 0.55 & 3.25 & 0.68 & 1.89 & 0.27 & 1.92 & 0.28 & 123.25 \\
\hline PAAS & 38.2 & 79.6 & 8.83 & 33.9 & 5.55 & 1.08 & 4.66 & 0.77 & 4.68 & 0.99 & 2.85 & 0.41 & 2.82 & 0.43 & 184.77 \\
\hline UCC & 30 & 64 & 7.10 & 26 & 4.5 & 0.88 & 3.8 & 0.64 & 3.50 & 0.80 & 2.30 & 0.33 & 2.20 & 0.32 & 146.37 \\
\hline
\end{tabular}


Table 4: Weathering parameters, major oxides and rare earth element ratios for the shale of Chia Gara Formation

\begin{tabular}{|c|c|c|c|c|c|c|c|c|c|c|c|c|}
\hline Sample no. & CIA & PIA & CIW & $\mathrm{Al}_{2} \mathrm{O}_{3} / \mathrm{TiO}_{2}$ & LREE/ HREE & $\Sigma$ REE & $\mathrm{Ce} / \mathrm{Ce}^{*}$ & $\mathrm{Eu} / \mathrm{Eu}^{*}$ & $(\mathrm{La} / \mathrm{Yb})_{\mathrm{n}}$ & $(\mathrm{La} / \mathrm{Sm})_{\mathrm{n}}$ & $(\mathrm{Gd} / \mathrm{Yb})_{\mathrm{n}}$ & $(\mathrm{La} / \mathrm{Lu})_{\mathrm{n}}$ \\
\hline & \multicolumn{12}{|c|}{ Barsarin section } \\
\hline CBN 1 & 86.44 & 98.76 & 98.94 & 18.56 & 9.75 & 118.73 & 1.07 & 0.69 & 10.54 & 3.61 & 1.65 & 10.73 \\
\hline CBN 2 & 86.29 & 95.96 & 96.43 & 17.18 & 9.27 & 120.95 & 0.91 & 0.68 & 11.26 & 4.21 & 1.60 & 10.96 \\
\hline CBN 3 & 78.83 & 88.67 & 90.32 & 17.65 & 7.26 & 93.52 & 0.84 & 0.62 & 7.97 & 3.66 & 1.37 & 10.24 \\
\hline CBN 4 & 71.53 & 77.43 & 80.15 & 15.30 & 7.27 & 79.58 & 0.81 & 0.66 & 8.96 & 4.05 & 1.50 & 10.44 \\
\hline CBN 5 & 69.81 & 74.52 & 77.22 & 16.18 & 8.42 & 101.92 & 0.96 & 0.74 & 9.07 & 3.58 & 1.55 & 9.75 \\
\hline CBN 6 & 74.40 & 80.15 & 82.24 & 16.21 & 8.57 & 118.42 & 1.01 & 0.61 & 9.44 & 3.17 & 1.75 & 10.79 \\
\hline CBN 7 & 72.54 & 77.57 & 79.83 & 15.44 & 9.22 & 96.23 & 0.98 & 0.67 & 9.46 & 3.89 & 1.45 & 10.90 \\
\hline CBN 8 & 82.77 & 91.08 & 92.09 & 15.77 & 8.43 & 194.43 & 1.01 & 0.66 & 9.52 & 3.40 & 1.74 & 10.13 \\
\hline CBN 9 & 86.36 & 95.68 & 96.17 & 13.93 & 8.25 & 148.91 & 0.97 & 0.66 & 8.45 & 4.06 & 1.23 & 8.56 \\
\hline CBN 10 & 90.17 & 98.75 & 98.87 & 16.06 & 7.74 & 92.52 & 1.01 & 0.72 & 7.94 & 3.47 & 1.63 & 8.49 \\
\hline CBN 11 & 89.68 & 99.16 & 99.25 & 14.71 & 7.80 & 116.5 & 0.98 & 0.69 & 8.44 & 3.56 & 1.41 & 8.31 \\
\hline CBN 12 & 88.29 & 98.03 & 98.25 & 15.70 & 8.53 & 113.28 & 0.95 & 0.59 & 9.49 & 4.25 & 1.49 & 9.53 \\
\hline CBN 13 & 89.73 & 99.02 & 99.12 & 15.21 & 8.89 & 134.75 & 0.96 & 0.71 & 9.33 & 3.91 & 1.32 & 9.77 \\
\hline CBN 14 & 88.97 & 98.22 & 98.41 & 14.56 & 8.56 & 116.02 & 0.97 & 0.70 & 9.10 & 3.95 & 1.52 & 11.07 \\
\hline CBN 15 & 92.63 & 98.69 & 98.78 & 15.90 & 8.86 & 144.51 & 0.97 & 0.69 & 9.75 & 3.79 & 1.51 & 10.83 \\
\hline CBN 16 & 92.13 & 98.32 & 98.43 & 15.77 & 9.98 & 179.16 & 0.98 & 0.66 & 10.35 & 4.60 & 1.26 & 10.66 \\
\hline CBN 17 & 93.55 & 98.33 & 98.42 & 17.08 & 11.28 & 163.14 & 0.99 & 0.66 & 11.44 & 4.94 & 1.26 & 13.53 \\
\hline CBN 18 & 93.93 & 98.82 & 98.88 & 16.81 & 10.36 & 153.17 & 1.01 & 0.60 & 12.07 & 4.29 & 1.59 & 11.35 \\
\hline CBN 19 & 94.87 & 99.44 & 99.47 & 17.25 & 10.45 & 157.24 & 0.99 & 0.64 & 11.76 & 4.03 & 1.61 & 11.52 \\
\hline CBN 20 & 94.91 & 99.39 & 99.42 & 16.21 & 9.53 & 133.04 & 0.97 & 0.64 & 10.92 & 4.04 & 1.61 & 11.50 \\
\hline CBN 21 & 93.13 & 98.01 & 98.11 & 18.59 & 10.30 & 185.28 & 0.98 & 0.68 & 11.21 & 4.59 & 1.44 & 12.70 \\
\hline \multirow[t]{2}{*}{ Average } & 86.24 & 93.52 & 94.23 & 16.19 & 9.04 & 131.48 & 0.97 & 0.66 & 9.86 & 3.97 & 1.49 & 10.50 \\
\hline & \multicolumn{12}{|c|}{ Banik section } \\
\hline CBK 1 & 85.47 & 99.16 & 99.30 & 20.62 & 10.76 & 177.06 & 1.03 & 0.64 & 11.22 & 4.17 & 1.38 & 11.53 \\
\hline CBK 2 & 86.70 & 99.17 & 99.29 & 19.17 & 10.14 & 39.78 & 1.05 & 0.67 & 10.14 & 4.80 & 1.38 & 15.57 \\
\hline CBK 3 & 88.52 & 99.21 & 99.31 & 19.67 & 10.07 & 196.41 & 1.01 & 0.64 & 10.36 & 3.97 & 1.48 & 10.31 \\
\hline CBK 4 & 76.32 & 97.89 & 98.51 & 22.60 & 8.85 & 103.63 & 0.96 & 0.73 & 9.86 & 4.07 & 1.62 & 10.81 \\
\hline CBK 5 & 74.09 & 95.54 & 96.91 & 20.15 & 9.70 & 78.93 & 0.89 & 0.76 & 11.20 & 4.67 & 1.53 & 11.54 \\
\hline CBK 6 & 69.19 & 93.49 & 96.02 & 23.62 & 9.50 & 111.5 & 0.91 & 0.72 & 10.79 & 4.29 & 1.64 & 13.34 \\
\hline CBK 7 & 61.85 & 83.13 & 91.11 & 21.29 & 9.40 & 85.98 & 0.91 & 0.75 & 10.55 & 3.94 & 1.54 & 12.40 \\
\hline CBK 8 & 60.68 & 74.44 & 84.45 & 18.22 & 7.50 & 109.65 & 0.87 & 0.63 & 8.65 & 3.73 & 1.46 & 8.14 \\
\hline CBK 9 & 69.75 & 96.67 & 98.02 & 17.60 & 8.59 & 75.48 & 0.86 & 0.60 & 9.61 & 4.41 & 1.34 & 9.94 \\
\hline CBK 10 & 73.01 & 97.34 & 98.26 & 17.19 & 8.48 & 114.56 & 0.89 & 0.68 & 9.17 & 4.20 & 1.40 & 11.33 \\
\hline CBK 11 & 74.97 & 98.04 & 98.66 & 17.00 & 8.45 & 129.92 & 0.89 & 0.65 & 9.78 & 4.38 & 1.53 & 9.77 \\
\hline CBK 12 & 89.05 & 99.76 & 99.79 & 16.51 & 8.48 & 182.48 & 0.97 & 0.65 & 8.89 & 3.80 & 1.52 & 9.66 \\
\hline CBK 13 & 89.69 & 98.50 & 98.65 & 15.55 & 7.89 & 76.02 & 0.86 & 0.70 & 8.69 & 3.65 & 1.47 & 9.34 \\
\hline CBK 14 & 92.88 & 99.12 & 99.18 & 15.66 & 8.27 & 127.68 & 0.85 & 0.70 & 8.58 & 4.07 & 1.20 & 9.34 \\
\hline Average & 78.01 & 95.10 & 96.96 & 18.91 & 8.98 & 114.94 & 0.93 & 0.67 & 9.75 & 4.08 & 1.46 & 10.49 \\
\hline Total avg. & 82.98 & 94.16 & 95.32 & 17.28 & 9.00 & 123.25 & 0.95 & 0.67 & 9.79 & 4.02 & 1.47 & 10.49 \\
\hline PAAS & & & & & 9.49 & 184.77 & 1.02 & 0.65 & 9.15 & 4.33 & 1.34 & 9.22 \\
\hline UCC & & & & & 9.54 & 146.37 & 1.03 & 0.65 & 9.21 & 4.20 & 1.40 & 9.73 \\
\hline
\end{tabular}




\section{DISCUSSION}

\section{Paleo-Weathering of the Source Area}

The intensity and degree of chemical weathering in clastic rocks can be evaluated by using the relationship between alkali and alkaline earth elements (Nesbitt and Young, 1982). In weathering section, the insoluble elements $(\mathrm{Al}, \mathrm{Ba}, \mathrm{Rb})$ are resistant, and labile elements $(\mathrm{Na}, \mathrm{Ca}$, and $\mathrm{Sr})$ are mainly leached from the profile (Nesbitt et al., 1980). These chemical changes are reflected in the sedimentary record (Wronkiewicz and Condie, 1987) supplying a useful tool for estimating source area weathering conditions. The degree of weathering can be quantified by calculating the chemical index of alteration (CIA), (Nesbitt and Young, 1982), plagioclase index of alteration (PIA; Fedo et al., 1995) and chemical index of weathering (CIW; Harnois, 1988). Nesbitt and Young (1984) used the ternary diagram (A-CN-K) considering $\mathrm{Al}_{2} \mathrm{O}_{3}-\left(\mathrm{CaO}+\mathrm{Na}_{2} \mathrm{O}\right)-\mathrm{K}_{2} \mathrm{O}$ to deduce weathering trends. The chemical index of alteration (CIA) was calculated with the formula:

CIA $=\left[\mathrm{Al}_{2} \mathrm{O}_{3} /\left(\mathrm{Al}_{2} \mathrm{O}_{3}+\mathrm{CaO} *+\mathrm{Na}_{2} \mathrm{O}+\mathrm{K}_{2} \mathrm{O}\right)\right] \times 100$ (Nesbitt and Young, 1982)

Where $\mathrm{CaO}^{*}$ is the $\mathrm{CaO}$ content incorporated in the silicate fraction of the studied samples (Fedo et al., 1995).

Since there is no direct method to quantify the contents of $\mathrm{CaO}$ belonging to silicate and non-silicate fractions, here we used the method suggested by McLennan et al. (1993) to calculate the $\mathrm{CaO}$ in silicate fraction; the molar proportion of $\mathrm{Na}_{2} \mathrm{O}$ is regarded as the molar proportion of $\mathrm{CaO}$ of the silicate fraction, when the $\mathrm{CaO}$ content was high. The CIA, PIA, and CIW values of $\sim$ 60 indicates low weathering, $\sim 60-80$ moderate weathering, and more than 80 indicate intensive weathering (Fedo et al., 1995). The calculated CIA values of the shale from Chia Gara for two sections vary between 60.68 to $94.91 \%$ (average $=82.98 \%$; Table 4 ). The result of PIA shows no significant difference between Barsarin (93.52) and Banik (95.10; Table 4) shales. This average is higher than the PAAS values (70-75; Taylor and McLennan, 1985), suggesting an intensive degree of chemical weathering in the source area (Fig. 7). The CIA values were plotted on A-CN$\mathrm{K}$ diagram (Fig. 7), to evaluate the mobility of the elements during the advance of chemical weathering. All the shale samples fall above the plagioclase-feldspar line and exhibit a definite trend. The samples of Banik section and some of Barsarin are clustered near the A-K line, towards the illite composition (Barsarin section) and the muscovite composition (Banik section), and do not indicate any clear-cut evidence of K-metasomatism or direct weathering back to the source (Fig. 7). The other samples of Barsarin section do not incline to $\mathrm{K}_{2} \mathrm{O}$-apex, the linear 
weathering trend of these shales intersects the plagioclase-K-feldspar joint and is sub parallel to the A-CN joint, indicating of relatively steady state weathering conditions and predicts a source rock with a plagioclase/K-feldspar ratio of about 5:1 (Fig. 7).

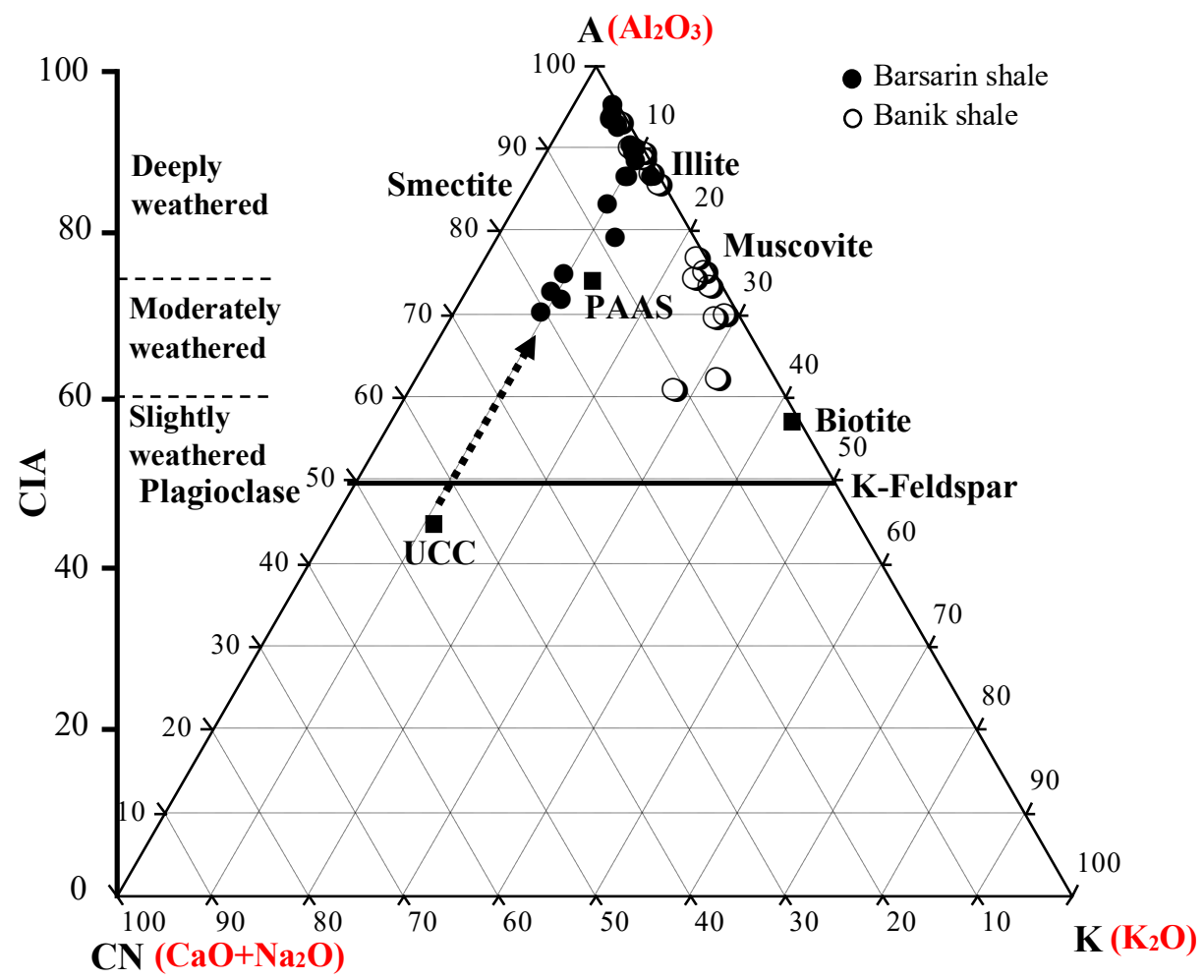

Fig. 7: A-CN-K ternary diagram in molecular proportions for the Chia Gara shales (after Nesbitt and Young, 1984). Also plotted is the average Upper Continental Crust (UCC) and Post Archean Australian Shale (PAAS) (Taylor and McLennan, 1985)

The A-CN-K diagram indicates that the samples were derived from the upper continental crust (UCC) influenced by intense chemical weathering (Madhavaraju et al. 2016). The samples that are concentrated nearly parallel to A-K line close to muscovite and illite composition, suggests the advance in weathering; the K- feldspar was destroyed by $\mathrm{K}$ leaching out and the residues were enriched with $\mathrm{Al}$ and therefore trend towards the A apex (Wouatong et al., 2013).

The quantitative measure of plagioclase weathering is estimated by calculating the PIA:

$\mathrm{PIA}=\left[\left(\mathrm{Al}_{2} \mathrm{O}_{3}-\mathrm{K}_{2} \mathrm{O}\right) /\left(\mathrm{Al}_{2} \mathrm{O}_{3}+\mathrm{CaO}^{*}+\mathrm{Na}_{2} \mathrm{O}-\mathrm{K}_{2} \mathrm{O}\right)\right] \times 100$ (Fedo et al., 1995)

The PIA values range from 74.44 to $99.76 \%$ (average= 94.16\%) indicating a high degree of alteration at the source area.

The chemical index of weathering was calculated with the formula: 
$\mathrm{CIW}=\left[\mathrm{Al}_{2} \mathrm{O}_{3} /\left(\mathrm{Al}_{2} \mathrm{O}_{3}+\mathrm{CaO}+\mathrm{Na}_{2} \mathrm{O}\right)\right] \times 100$ (Harnois, 1988)

The CIW values vary from 77.22 to $99.47 \%$ (average $=95.32 \%$; Table 4 ). The PIA values of the studied shale are comparable to the calculated CIW values (Harnois, 1988) this does not include $\mathrm{K}_{2} \mathrm{O}$. Based on CIA, PIA, and CIW values $(>80 \%)$, it can be concluded that the lithocomponents in the shale were subjected to intense chemical weathering and these results support the humid paleoclimate conditions in the source area during Late Jurassic-Early Cretaceous.

\section{Provenance}

The chemical analyses of the major, trace and rare earth elements of the clastic sediments were widely used to distinguish the source rock identity (Armstrong-Altrin et al., 2015; Tobia and Shangola, 2016; Tobia and Mustafa, 2016; Zaid, 2017). In order to deduce the provenance of the rocks, several discrimination diagrams were proposed based on major and trace elements (Roser and Korsch, 1988; Floyd et al., 1989; McLennan et al., 1993; Verma and ArmstrongAltrin, 2013, 2016; Verma et al., 2016).

The ratios of $\mathrm{Al}_{2} \mathrm{O}_{3} / \mathrm{TiO}_{2}$ in clastic sedimentary rocks are regarded as a powerful tool to distinguish the types of source rocks (Garcia et al., 1994; Andersson et al., 2004), since the ratio $<14$ in sediments is indicative of mafic source rocks, whereas ratios ranging from 19 to 28 reveal felsic source rocks. $\mathrm{Al}_{2} \mathrm{O}_{3} / \mathrm{TiO}_{2}$ ratios in the shale of the Chia Gara Formation, which range from 13.93 to 23.62 (Table 4), suggest that these sediments are derived from felsic source rocks.

The ratio of $\mathrm{Th} / \mathrm{Sc}$ is a dependable tool for source composition and the discrimination between felsic and mafic source components (McLennan et al., 1990; McLennan and Taylor, 1991). Thorium is highly incompatible and common in crustal sources. Thus, the $\mathrm{Th} / \mathrm{Sc}$ ratio will be high in the rocks derived from the earths crust and low in that derived from the mantle. The Chia Gara samples show $\mathrm{Th} / \mathrm{Sc}$ ratios between 0.79 and 1.42 (average $=1.04$ ) for Barsaren section and between 0.72 and 0.98 (average $=0.84$ ) for Banik section. For Chia Gara (0.96) as a whole, the Th/Sc ratio is nearly similar to PAAS (0.91; Taylor and McLennan, 1985) and higher than of UCC (0.79; McLennan, 2001).

$\mathrm{La} / \mathrm{Th}$ ratios are useful indicators for providing source area composition, while Hf reveals the degree of recycling. The average La/Th values of the shale from Barsarin and Banik sections are 3.39 and 3.49, respectively (Table 5). The samples show higher values relative to PAAS and UCC (2.61 and 2.80, respectively). On a La/Th vs. Hf plot (Floyd and Leveridge, 1987; Spalletti et al. 2012), the Chia Gara shale samples are scattered in the felsic and toward the andesitic fields (Fig. 8). 


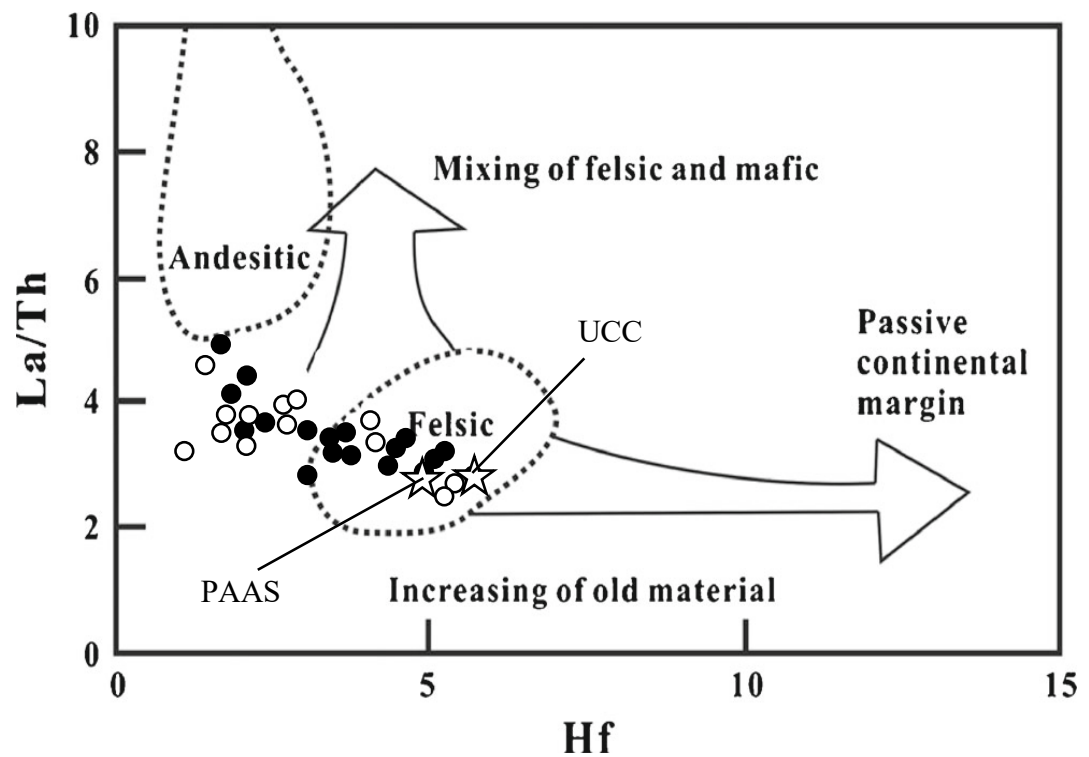

Fig. 8: La/Th vs Hf diagram for the shale of Chia Gara Formation (fields after Floyd and Leveridge, 1987)

The REEs, $\mathrm{Sc}, \mathrm{Co}$, Th, and $\mathrm{Cr}$ are employed to predict the composition of the source area (Taylor and McLennan, 1985; McLennan and Taylor, 1991). These elements have very short residence times in seawater and are transferred into the sedimentary record. This represents compatible ( $\mathrm{Sc}$ and $\mathrm{Co}$ ) and incompatible elements (REEs and $\mathrm{Th}$ ), and ratios that are useful in discriminating between mafic and felsic source rocks. However, the ratios $\mathrm{La} / \mathrm{Sc}, \mathrm{La} / \mathrm{Co}, \mathrm{Th} / \mathrm{Sc}$, $\mathrm{Th} / \mathrm{Co}, \mathrm{Cr} / \mathrm{Th},(\mathrm{La} / \mathrm{Lu})_{\mathrm{cn}}$ and $\mathrm{Eu} / \mathrm{Eu}^{*}{ }_{\mathrm{cn}}$, are significantly different in mafic and felsic rocks and may constraints on the provenance composition (Wronkiewicz and Condie, 1990; Cox et al., 1995; Cullers, 1995; Asiedu et al., 2004; Dai et al., 2016). The ratios of the Chia Gara shales are compared with those of sediments derived from felsic and mafic rocks as well as with UCC and PAAS values (Table 6). These ratios for Barsarin are higher than that for Banik shale (except $\mathrm{Cr} / \mathrm{Th}$ ), and the comparison suggests these ratios are within the range of felsic rocks. 
Table 5: Trace element ratios for the shale of Chia Gara Formation

\begin{tabular}{|c|c|c|c|c|c|c|c|c|c|c|c|c|c|c|}
\hline Sample no. & $\mathrm{La} / \mathrm{Sc}$ & $\mathrm{La} / \mathrm{Th}$ & $\mathrm{La} / \mathrm{Co}$ & $\mathrm{Zr} / \mathrm{Sc}$ & $\mathrm{Th} / \mathrm{Sc}$ & $\mathrm{Zr} / \mathrm{Hf}$ & $\mathrm{Th} / \mathrm{U}$ & $\mathrm{Cr} / \mathrm{Th}$ & $\mathrm{Ti} / \mathrm{Zr}$ & $\mathrm{U} / \mathrm{Th}$ & $\mathrm{V} / \mathrm{Cr}$ & $\mathrm{V} / \mathrm{Sc}$ & $\mathrm{Ni} / \mathrm{Co}$ & $\mathrm{U}-\mathrm{Th} / 3$ \\
\hline & \multicolumn{14}{|c|}{ Barsarin section } \\
\hline CBN 1 & 2.25 & 2.80 & 0.38 & 12.00 & 0.81 & 44.00 & 0.17 & 10.16 & 29.97 & 5.80 & 6.88 & 56.27 & 4.35 & 48.45 \\
\hline CBN 2 & 4.07 & 3.90 & 1.90 & 15.71 & 1.04 & 39.29 & 0.32 & 12.31 & 31.06 & 3.12 & 7.60 & 97.71 & 14.13 & 20.36 \\
\hline CBN 3 & 3.62 & 4.31 & 3.10 & 14.33 & 0.84 & 43.00 & 0.27 & 19.84 & 27.88 & 3.71 & 9.08 & 151.33 & 33.43 & 17.02 \\
\hline CBN 4 & 3.82 & 4.85 & 3.82 & 14.00 & 0.79 & 43.75 & 0.23 & 27.92 & 28.26 & 4.38 & 5.45 & 120.00 & 43.40 & 15.94 \\
\hline CBN 5 & 3.60 & 4.03 & 1.35 & 13.50 & 0.89 & 45.00 & 0.36 & 13.06 & 29.60 & 2.80 & 4.10 & 47.83 & 10.38 & 13.21 \\
\hline CBN 6 & 2.99 & 3.57 & 2.66 & 12.50 & 0.84 & 43.48 & 0.41 & 11.96 & 28.17 & 2.44 & 3.69 & 36.88 & 15.56 & 14.12 \\
\hline CBN 7 & 3.50 & 3.27 & 2.63 & 15.17 & 1.07 & 45.50 & 0.67 & 12.44 & 28.32 & 1.49 & 5.78 & 77.00 & 30.13 & 7.45 \\
\hline CBN 8 & 3.15 & 3.39 & 2.73 & 13.00 & 0.93 & 36.74 & 1.12 & 12.40 & 30.15 & 0.90 & 3.76 & 43.38 & 14.93 & 6.82 \\
\hline CBN 9 & 3.00 & 3.11 & 3.67 & 18.45 & 0.96 & 39.04 & 1.07 & 13.21 & 26.28 & 0.94 & 5.18 & 65.91 & 20.00 & 6.40 \\
\hline CBN 10 & 3.76 & 3.57 & 3.13 & 18.40 & 1.05 & 46.00 & 0.47 & 11.41 & 23.45 & 2.15 & 4.78 & 57.40 & 13.50 & 9.55 \\
\hline CBN 11 & 3.20 & 3.32 & 4.27 & 16.38 & 0.96 & 39.70 & 0.59 & 10.39 & 27.00 & 1.69 & 6.03 & 60.25 & 27.17 & 10.43 \\
\hline CBN 12 & 3.21 & 3.41 & 3.21 & 17.38 & 0.94 & 38.61 & 0.52 & 11.94 & 25.44 & 1.91 & 4.89 & 55.00 & 19.63 & 11.89 \\
\hline CBN 13 & 3.01 & 3.07 & 3.76 & 16.30 & 0.98 & 44.05 & 0.84 & 11.22 & 26.84 & 1.19 & 5.81 & 63.90 & 28.25 & 8.38 \\
\hline $\mathrm{CBN} 14$ & 3.66 & 3.46 & 3.66 & 17.86 & 1.06 & 41.67 & 1.02 & 10.83 & 26.85 & 0.98 & 6.03 & 68.86 & 14.29 & 4.77 \\
\hline CBN 15 & 3.13 & 3.14 & 3.48 & 15.30 & 1.00 & 41.35 & 0.68 & 9.04 & 26.25 & 1.46 & 6.36 & 57.20 & 23.33 & 11.23 \\
\hline CBN 16 & 3.51 & 2.76 & 3.01 & 19.58 & 1.27 & 41.96 & 1.97 & 9.18 & 26.78 & 0.51 & 4.84 & 56.42 & 13.50 & 2.68 \\
\hline CBN 17 & 3.91 & 2.92 & 3.91 & 18.30 & 1.34 & 37.35 & 1.80 & 7.46 & 27.51 & 0.56 & 5.00 & 50.00 & 11.60 & 2.97 \\
\hline CBN 18 & 3.89 & 2.97 & 5.00 & 18.44 & 1.31 & 38.60 & 1.09 & 7.63 & 24.91 & 0.92 & 4.97 & 49.67 & 19.71 & 6.87 \\
\hline CBN 19 & 3.94 & 3.11 & 4.44 & 17.89 & 1.27 & 36.59 & 1.11 & 8.77 & 26.43 & 0.90 & 3.68 & 40.89 & 17.38 & 6.50 \\
\hline CBN 20 & 3.32 & 3.15 & 3.32 & 14.78 & 1.06 & 39.12 & 1.49 & 8.42 & 31.55 & 0.67 & 4.71 & 41.89 & 11.67 & 3.19 \\
\hline CBN 21 & 4.28 & 3.02 & 4.28 & 19.50 & 1.42 & 39.00 & 1.77 & 13.43 & 28.28 & 0.56 & 3.18 & 60.40 & 10.80 & 3.26 \\
\hline \multirow[t]{2}{*}{ Average } & 3.47 & 3.39 & 3.22 & 16.13 & 1.04 & 41.13 & 0.86 & 12.05 & 27.60 & 1.56 & 5.20 & 61.05 & 14.42 & 11.02 \\
\hline & \multicolumn{14}{|c|}{ Banik section } \\
\hline CBK 1 & 2.22 & 2.45 & 0.87 & 11.11 & 0.91 & 38.46 & 0.80 & 9.20 & 33.87 & 1.26 & 4.57 & 38.11 & 4.28 & 15.07 \\
\hline CBK 2 & 2.25 & 3.15 & 1.13 & 11.25 & 0.72 & 45.00 & 0.36 & 10.49 & 31.97 & 2.79 & 4.13 & 31.00 & 5.88 & 7.04 \\
\hline CBK 3 & 2.43 & 2.68 & 1.32 & 11.89 & 0.91 & 39.63 & 0.79 & 9.20 & 33.61 & 1.27 & 2.95 & 24.56 & 5.73 & 15.27 \\
\hline CBK 4 & 2.29 & 3.25 & 1.53 & 9.10 & 0.70 & 45.50 & 0.22 & 12.78 & 31.62 & 4.53 & 7.74 & 69.70 & 14.13 & 29.55 \\
\hline CBK 5 & 2.70 & 3.61 & 1.89 & 13.86 & 0.75 & 46.19 & 0.18 & 19.08 & 25.34 & 5.65 & 6.51 & 93.00 & 16.80 & 27.85 \\
\hline CBK 6 & 2.86 & 3.64 & 1.84 & 9.89 & 0.78 & 42.38 & 0.28 & 15.58 & 31.65 & 3.58 & 6.60 & 80.67 & 16.64 & 22.95 \\
\hline CBK 7 & 3.38 & 3.45 & 2.03 & 11.83 & 0.98 & 44.38 & 0.40 & 18.68 & 32.08 & 2.50 & 3.89 & 71.33 & 24.30 & 12.79 \\
\hline CBK 8 & 3.14 & 3.60 & 1.93 & 14.63 & 0.87 & 43.33 & 0.31 & 24.39 & 26.13 & 3.19 & 6.00 & 127.50 & 30.77 & 19.88 \\
\hline CBK 9 & 3.03 & 3.73 & 3.64 & 12.33 & 0.81 & 43.53 & 0.33 & 20.49 & 29.97 & 3.05 & 5.21 & 86.83 & 39.80 & 13.27 \\
\hline CBK 10 & 3.28 & 3.86 & 2.38 & 14.25 & 0.85 & 42.22 & 0.38 & 17.67 & 28.39 & 2.62 & 4.35 & 65.25 & 24.45 & 15.54 \\
\hline CBK 11 & 3.76 & 3.94 & 1.25 & 14.63 & 0.96 & 41.79 & 0.37 & 18.32 & 29.20 & 2.67 & 4.66 & 81.50 & 16.00 & 17.85 \\
\hline CBK 12 & 3.08 & 3.35 & 2.11 & 13.62 & 0.92 & 43.17 & 0.70 & 17.57 & 31.49 & 1.43 & 5.24 & 84.62 & 20.68 & 13.07 \\
\hline CBK 13 & 3.42 & 4.54 & 2.85 & 14.00 & 0.75 & 50.00 & 0.43 & 21.22 & 26.54 & 2.31 & 5.38 & 86.00 & 21.50 & 7.43 \\
\hline CBK 14 & 3.06 & 3.64 & 4.37 & 16.20 & 0.84 & 40.50 & 0.80 & 33.33 & 28.12 & 1.24 & 1.36 & 38.10 & 16.86 & 7.65 \\
\hline Average & 2.92 & 3.49 & 2.08 & 12.76 & 0.84 & 43.29 & 0.45 & 17.71 & 30.23 & 2.37 & 4.57 & 64.41 & 14.37 & 16.09 \\
\hline Total avg. & 3.25 & 3.43 & 2.77 & 14.78 & 0.96 & 42.00 & 0.70 & 14.32 & 29.03 & 1.93 & 4.84 & 62.79 & 14.39 & 13.05 \\
\hline PAAS & & 2.61 & & & 0.91 & & & & 28.26 & 0.21 & 1.36 & 9.38 & 2.39 & \\
\hline $\mathrm{UCC}$ & & 2.80 & & & 0.79 & & & & 15.77 & 0.26 & 1.71 & 5.45 & 2.00 & \\
\hline
\end{tabular}


Table 6: Elemental ratios for the shale from Chia Gara Formation compared with those of fine-fractions derived from felsic and mafic source rocks

\begin{tabular}{|c|c|c|c|c|c|c|c|}
\hline \multirow[t]{2}{*}{ Elemental ratio } & \multicolumn{2}{|c|}{ Range of sediments } & \multirow[t]{2}{*}{$\mathrm{UCC}^{2}$} & \multirow[t]{2}{*}{ PAAS $^{2}$} & \multicolumn{3}{|c|}{ Average of studied shale } \\
\hline & Felsic rocks $^{1}$ & Mafic rocks ${ }^{1}$ & & & Barsarin $^{3}$ & Banik $^{3}$ & Chia Gara ${ }^{3}$ \\
\hline $\mathrm{La} / \mathrm{Sc}$ & $2.5-16.3$ & $0.43-0.86$ & 2.21 & 2.4 & 3.47 & 2.92 & 3.25 \\
\hline $\mathrm{La} / \mathrm{Co}$ & $1.8-13.8$ & $0.14-0.38$ & 1.76 & 1.66 & 3.22 & 2.08 & 2.77 \\
\hline $\mathrm{Th} / \mathrm{Sc}$ & $0.84-20.5$ & $0.05-0.22$ & 0.79 & 0.90 & 1.04 & 0.84 & 0.96 \\
\hline $\mathrm{Th} / \mathrm{Co}$ & $0.67-19.4$ & $0.04-1.4$ & 0.63 & 0.63 & 0.98 & 0.59 & 0.82 \\
\hline $\mathrm{Cr} / \mathrm{Th}$ & $4.0-15.0$ & $25-50$ & 7.76 & 7.53 & 12.05 & 17.7 & 14.32 \\
\hline$(\mathrm{La} / \mathrm{Lu}) \mathrm{n}$ & $3.0-27$ & $1.10-7$ & 9.73 & & 10.93 & 10.56 & 10.71 \\
\hline $\mathrm{Eu} / \mathrm{Eu}^{*}$ & $0.4-0.94$ & $0.71-0.95$ & 0.63 & 0.63 & 0.67 & 0.68 & 0.67 \\
\hline
\end{tabular}

(1) Cullers (1994, 2000), Cullers and Podkovyrov (2000); (2) Taylor and McLennan (1985); ( $\left.{ }^{3}\right)$ This study

According to the $\mathrm{Th} / \mathrm{Sc}-\mathrm{Zr} / \mathrm{Sc}$ diagram (Fig. 9), Chia Gara samples are clustered between granite and andesite rocks along with the magmatic compositional variation trend of rocks, which indicates the mixed source rocks between felsic and intermediate.

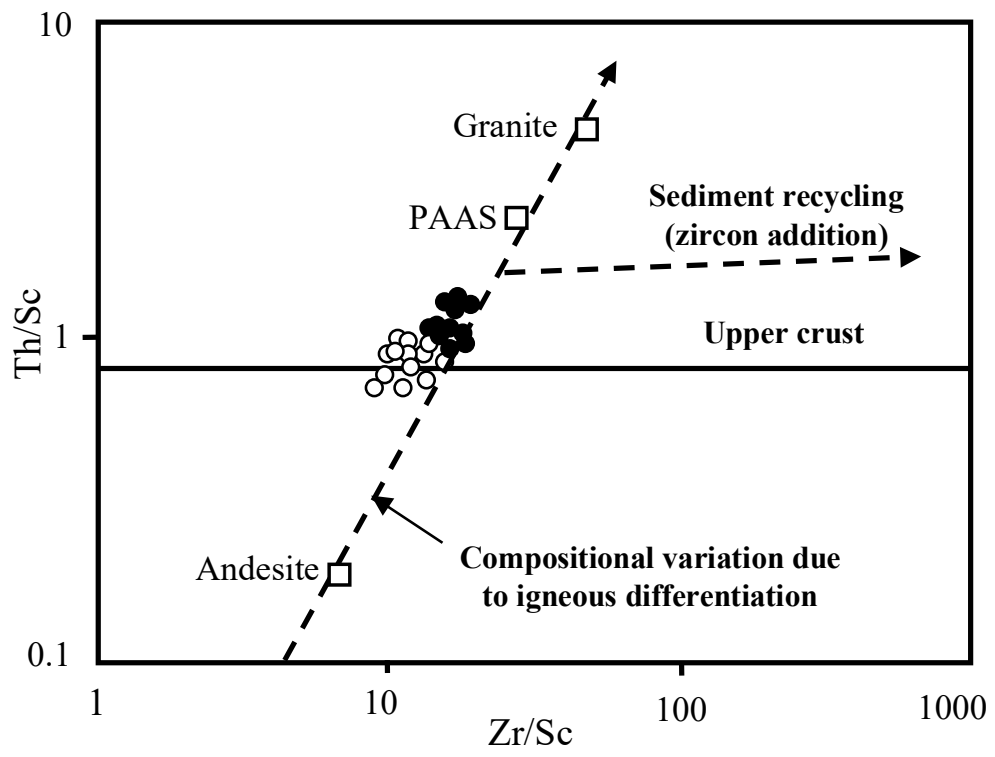

Fig. 9: Th/Sc vs. Zr/Sc provenance and recycling discrimination plot (after McLennan et al., 1993) for the shale of Chia Gara Formation. Average source rock compositions are of Proterozoic age (after Condie, 1993) 
Rare earth elements of sedimentary rocks can provide an important clue to unravel the source rock signatures (Bhatia and Crook, 1986; Gu et al., 2002; Armstrong-Altrin et al., 2013), as these elements have weak activity and are difficult to dissolve in water. Therefore, they can reflect source-area information. Mafic rocks commonly have low $(\mathrm{La} / \mathrm{Yb})_{\mathrm{cn}}$ ratio and do not have Eu anomaly, while silicic rocks commonly have higher $(\mathrm{La} / \mathrm{Yb})_{\mathrm{cn}}$ ratio and $\mathrm{Eu}$ anomaly (Cullers et al., 1997). The Chia Gara shales show LREE enrichment $\left((\mathrm{La} / \mathrm{Yb})_{\mathrm{cn}}=7.94-11.76\right.$, average $=$ 9.79) and relatively flat HREE pattern $\left((\mathrm{Gd} / \mathrm{Yb})_{\mathrm{cn}}=1.20-1.75\right.$, average $\left.=1.47\right)$, and clear negative $\left(\left(\mathrm{Eu} / \mathrm{Eu}^{*}=0.59-0.76\right.\right.$, average $=0.67$; Table 4$)$. Based on the aforementioned analysis, it can be summarized that REE characteristics indicate a mixture of felsic and intermediate source rocks.

\section{Geochemical Parameters Indicative of Redox Conditions}

The redox-sensitive elements are considered as a useful tool for recognizing the deposition of the sediments in non-marine and marine environments. These multi valence elements such as $\mathrm{V}, \mathrm{U}$, $\mathrm{Ni}, \mathrm{Cr}$, and Mo, their mobilization, precipitation, and concentration are mainly controlled by redox conditions and are enriched in anoxic sediments (Dypvik, 1984; Yarincik et al., 2000; Yang et al., 2004; Guo et al., 2007). Also, the ratios of these elements (U/Th, V/Cr, Ni/Co, and $\mathrm{V} / \mathrm{Sc}$ ) have been used to estimate the paleoredox conditions (Hatch and Leventhal, 1992; Jones and Manning, 1994; Pattan et al., 2005; Deepulal et al., 2012; Akinyemi et al. 2013; ArmstrongAltrin et al. 2015; Tobia and Shangola, 2016; Ramos-Vazquez et al., 2017; Anaya-Gregorio et al., 2018). The combined use of trace elements and their ratios may allow distinguishing the various depositional environments (oxic, suboxic, and anoxic).

The authigenic $U$ was used as procurator of redox conditions in marine sediments because it preserved in the oxygenated water. Meanwhile, becomes enriched in anoxic environment (Ramos-Vazquez et al., 2017; Anaya-Gregorio et al., 2018). Authigenic U was intended from the formula $=U_{\text {total }}-(\mathrm{Th} / 3)$. The sediments of high authigenic $U$ contents incline to be formed under anoxic conditions which allow great amounts of organic matter to accumulate and fixed (Wignall and Myers, 1988; Deepulal et al., 2012). Jones and Manning (1994) suggest less than 5ppm of authigenic $U$ to be indicative of oxic conditions. The shales of Chia Gara Formation are enriched in authigenic $U(13.05 \mathrm{ppm})$ (Table 5 and Fig. 10), which reflect anoxic environment for the studied shales.

$\mathrm{U} / \mathrm{Th}$ is considered as a reliable tool to infer the oxygenation condition in the basin of deposition (Dypvik, 1984; Madhavaraju and Ramasamy, 1999; Jones and Manning, 1994; Rimmer, 2004; Nagarajan et al., 2007a; Madhavaraju and Gonzalez- Leon, 2012; 

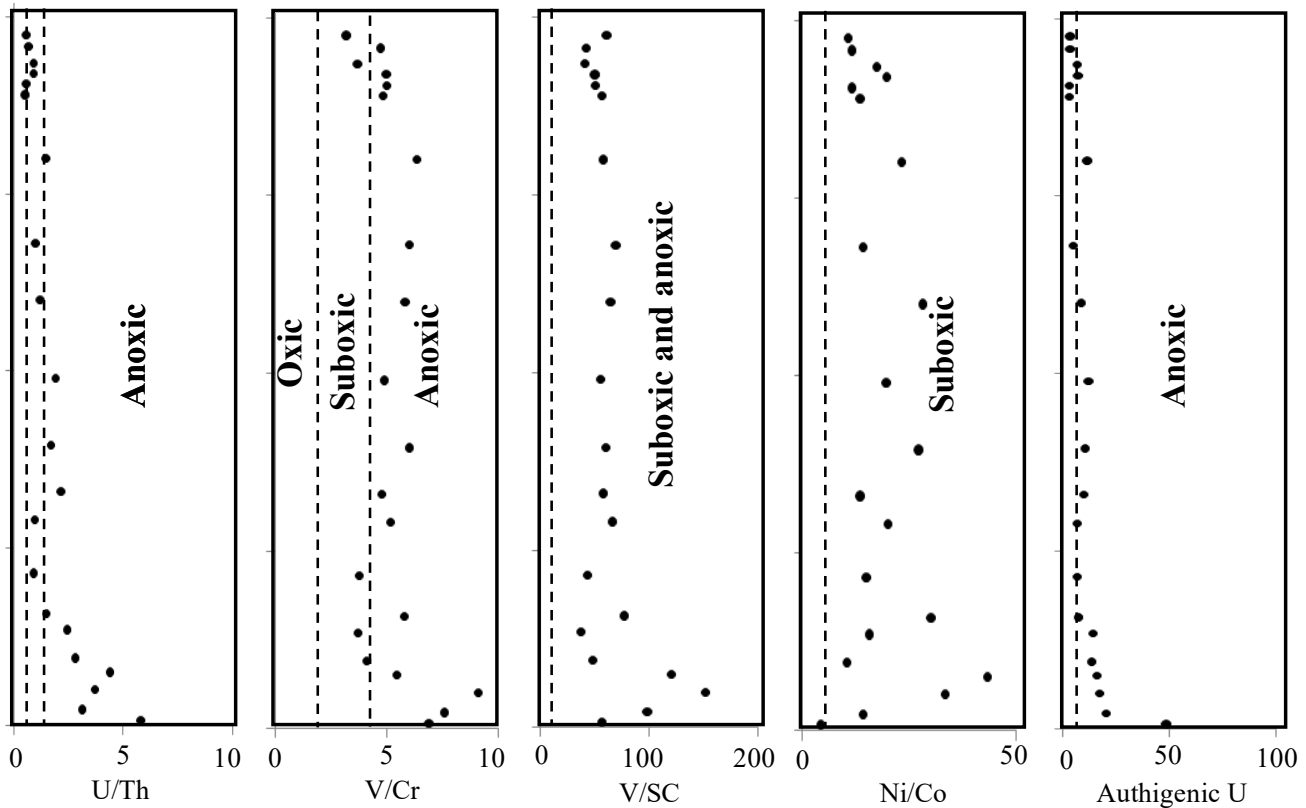

\section{Barsarin section}
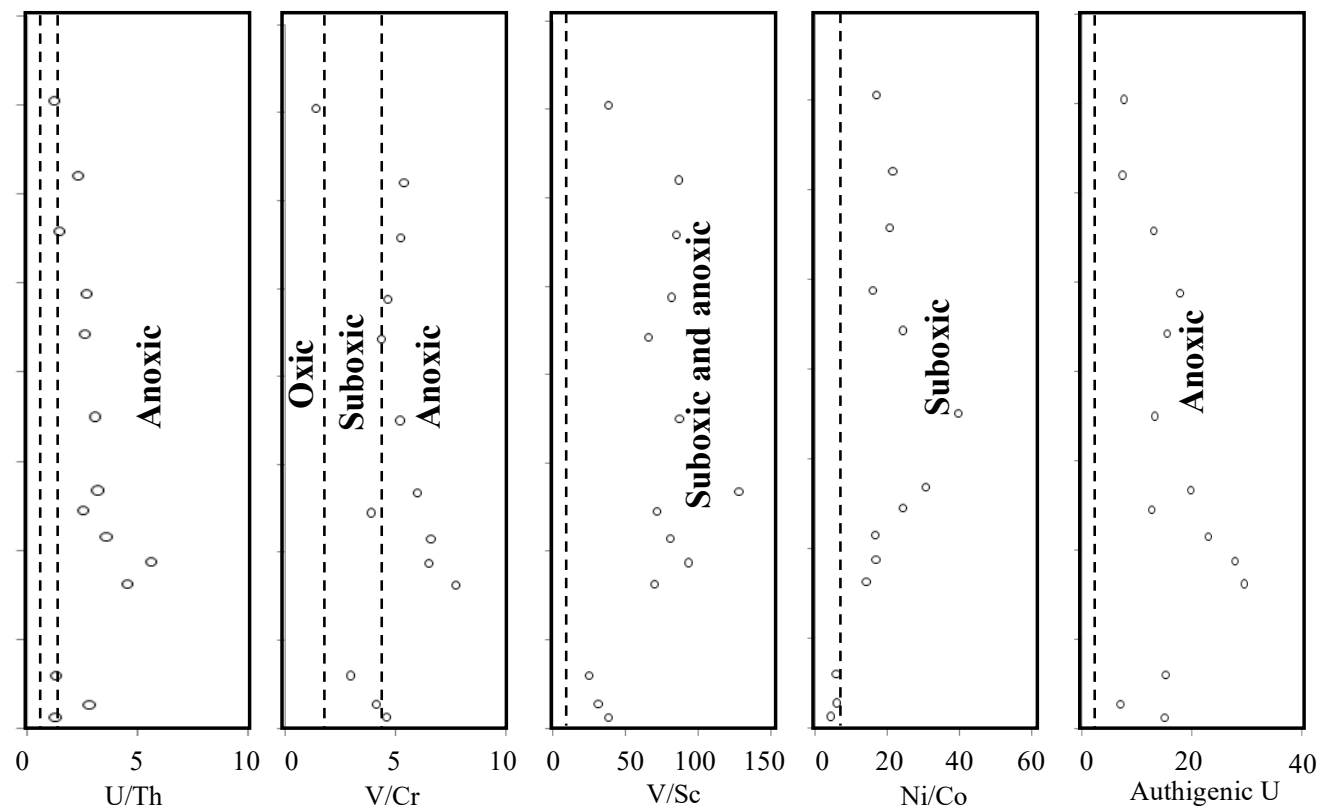

\section{Banik section}

Fig. 10: Chemostratigraphic profiles of redox-sensitive trace element ratios for the shales of Chia Gara Formation (after McKirdy and Hall, 2011)

Madhavaraju et al., 2016). The U/Th ratio can be regarded as a redox index, with high values $(>1.25)$ associated anoxic environment and low values $(<0.75)$ associated oxic environment (Jones and Manning, 1994; McKirdy et al., 2011). The high U/Th value of the Chia 
Gara shales (1.56 for Barsarin section and 2.37 for Banik section; Table 5) is indicative of the anoxic environment (except the upper part of the formation have more oxygenation conditions).

The $\mathrm{V} / \mathrm{Cr}$ values of the sediment can be also considered as a bottom water oxygenation index (Dill, 1986; Nagarajan et al., 2007a; Akinyemi et al., 2013). Bjorlykke (1974) reported the incorporation of $\mathrm{Cr}$ in the detrital fraction of sediments and its possible substitution for $\mathrm{Al}$ in the clay structure. Vanadium may be bound to organic matter by the incorporation of $\mathrm{V}^{4+}$ into porphyrins, and is generally found in sediments deposited in reducing environments (Shaw et al., 1990). The $\mathrm{V} / \mathrm{Cr}$ ratios more than 2 suggest anoxic conditions, whereas values less than 2 indicate more oxidizing conditions (Jones and Manning, 1994). The V/Cr ratios for the Chia Gara shales are more than 2 (5.20 for Barsarin, 4.57 for Banik, and 4.84 for average Chia Gara shale; Table 5). Accordingly, the Chia Gara shales are accumulated under anoxic conditions.

Kimura and Watanabe (2001) proposed V/Sc as a proxy indicator, and they suggested that $\mathrm{V} / \mathrm{Sc}$ ratios below 9 indicate oxidizing conditions and more than 9 is suboxic. The shales are characterized by the elevated values of V/Sc (61.05 for Barsarin, 64.41 for Banik, 62.79 for the average of Chia Gara); and are higher than PAAS (9.38) and UCC (5.45) as shown in Table 5. Therefore, the shales of Chia Gara Formation are accumulated in suboxic conditions (Fig. 10).

The Ni/Co ratios are a powerful tool for paleoredox conditions (Dypvik, 1984; Dill, 1986; Jones and Manning, 1994; Nagarajan et al., 2007a; Deepulal et al., 2012). The values of Ni/Co ratios less than 5 suggest oxic environments, whereas the values more than 5 indicate suboxic and anoxic environment of deposition (Jones and Manning, 1994). The Chia Gara shales have high Ni/Co values (14.42 for Barsarin, 14.37 for Banik, 14.39 for the average of Chia Gara shales; Table 5). These elevated values suggest that the deposition of the shales was established under anoxic conditions.

The studied parameters (authigenic $\mathrm{U}, \mathrm{U} / \mathrm{Th}, \mathrm{V} / \mathrm{Cr}, \mathrm{V} / \mathrm{Sc}$, and $\mathrm{Ni} / \mathrm{Co}$ ) strongly imply that the shales of Chia Gara Formation were accumulated under anoxic environment; with slightly more oxygenated at the upper part of the formation.

The $\mathrm{Al}_{2} \mathrm{O}_{3}, \mathrm{P}_{2} \mathrm{O}_{5}$, and $\mathrm{V}$ contents can be employed in the discrimination of the depositional environments for the mudstones. The $\mathrm{V}$ concentration is somewhat lower in freshwaters than marine deposits. The $\mathrm{P}_{2} \mathrm{O}_{5}$ contents considerably vary in seawater and controlled by many factors such as depth and temperature of the water (Dhannoun and Al-Dlemi, 2013). Figure (11a) shows $\mathrm{V}$ vs. $\mathrm{Al}_{2} \mathrm{O}_{3}$ plots of the Chia Gara shale, and the shallow marine and freshwater shales are distinguished from the deep marine one. The deep marine environment for the studied shale is 
consistent with the above paleoredox conditions (suboxic to anoxic). Figure (11b) shows $\mathrm{P}_{2} \mathrm{O}_{5}$ vs. $\mathrm{Al}_{2} \mathrm{O}_{3}$ plot of the Chia Gara samples. In this diagram, most of the samples fall in the deep depositional environments and the shale of Banik section is deeper than that of Barsarin. This interpretation has confirmed the results of $\mathrm{V}$ vs. $\mathrm{Al}_{2} \mathrm{O}_{3}$. These sediments were deposited in the deep marine environment, where the $\mathrm{P}$ content was high as a result of high biologic products.
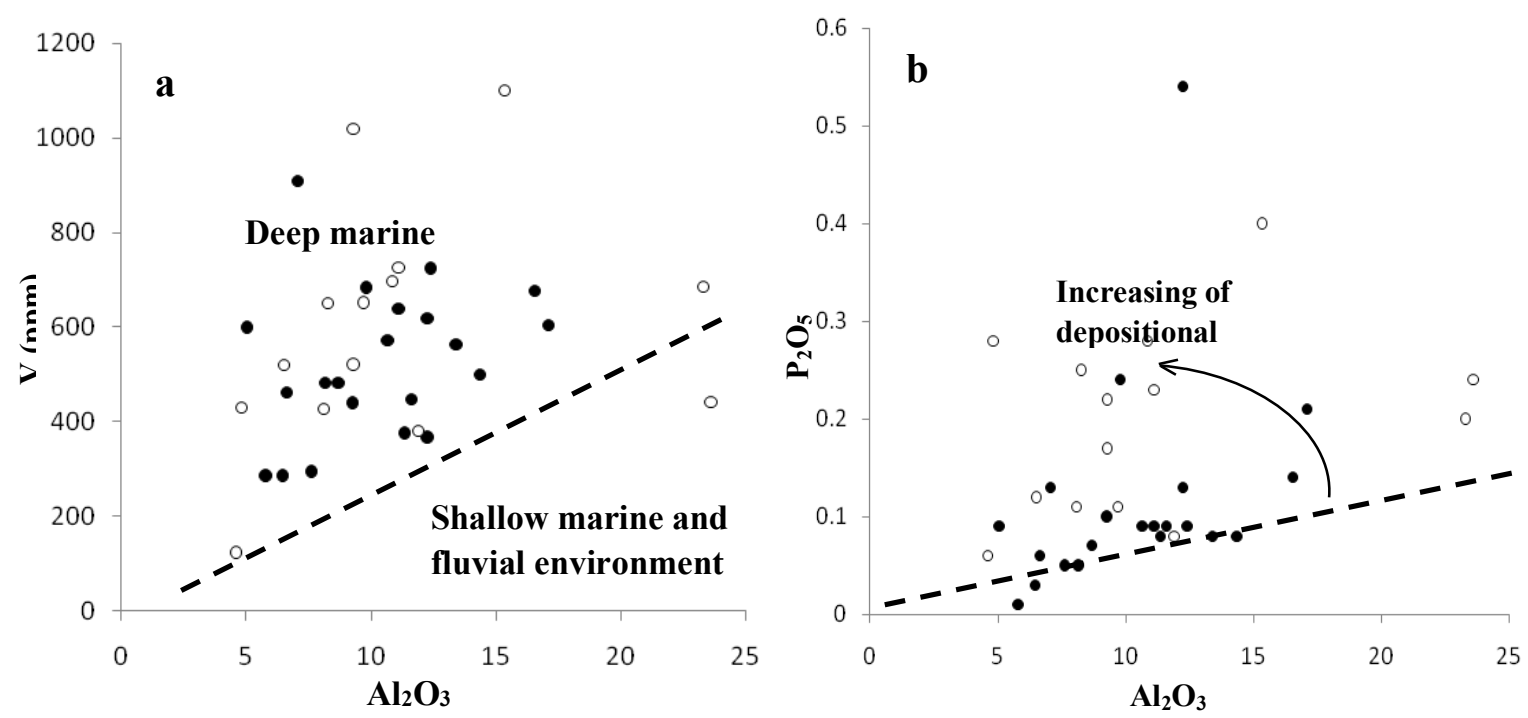

Fig. 11: Plots of (a) $\mathrm{Al}_{2} \mathrm{O}_{3}$ vs. $\mathrm{V}$ and and (b) $\mathrm{Al}_{2} \mathrm{O}_{3}$ vs. $\mathrm{P}_{2} \mathrm{O}_{5}$ for the shale from Chia Gara Formation for paleoenvironmental reconstruction (after Mortazavi et al., 2013)

\section{CONCLUSIONS}

The shale of Chia Gara Formation shows high $\mathrm{CaO}$ content that causes a high dilution effect on the other major, trace, and rare earth elements; and is enriched in $\mathrm{Sr}, \mathrm{U}, \mathrm{V}, \mathrm{Ni}$ and depleted in $\mathrm{Rb}$, $\mathrm{Ba}$, Th, Y, Zr, Nb, Hf, Sc, and Co. Chondrite normalized REE patterns show enrichment in LREE and depletion HREE in addition to the nearly flat HREE pattern, with negative Eu anomalies and moderate fractionation between LREE and HREE. Geochemical parameters (CIA, $\mathrm{CIW}$, and PIA) and A-CN-K diagram, reveal intense chemical weathering in the source area. The shale of Barsarin area was subject to more intense chemical weathering than at Banik. The ratios $\left(\mathrm{Al}_{2} \mathrm{O}_{3} / \mathrm{TiO}_{2}, \mathrm{Th} / \mathrm{Sc}, \mathrm{La} / \mathrm{Th}, \mathrm{La} / \mathrm{Sc}, \mathrm{La} / \mathrm{Co}, \mathrm{Th} / \mathrm{Co}, \mathrm{Cr} / \mathrm{Th},(\mathrm{La} / \mathrm{Lu})_{\mathrm{cn}}\right.$ and $\left.\mathrm{Eu} / \mathrm{Eu}{ }_{\mathrm{cn}}\right)$, and the diagrams (Th/Sc-Zr/Sc and La/Th-Hf) suggest a mixing source of felsic and intermediate rocks. The felsic rocks are derived from the Rutba Uplift or/and Mosul High which were a positive area at the time of deposition; and the intermediate rocks may derive from the volcanic material during the spreading of Southern Neo-Tethys Ocean. The authigenic uranium and U/Th, V/Cr, 
$\mathrm{Ni} / \mathrm{Co}$, and $\mathrm{V} / \mathrm{Sc}$ ratios refer suboxic to anoxic deep marine environment of deposition for the shale of Chia Gara Formation.

\section{REFERENCES}

Absar, N., Raza, M., Roy, M., Naqvi, S.M., and Roy, A.K., 2009. Composition and weathering conditions of palaeoproterozoic upper crust of Bundelkhand Craton, Central India: records from the geochemistry of clastic sediments of 1.9 Gwalior Group. Precamb Res, 168: 313 - 329.

Akinyemi, S.A., Adebayo, O.F., Ojo, O.A., Fadipe, O.A., and Gitari, W.M., 2013. Mineralogy and geochemical appraisal of paleo-redox indicators in Maastrichtian outcrop shales of Mamu formation, Anambra Basin, Nigeria. J Natur Sci Res, 3: $48-64$.

Al-Ameri, T.K., and Al-Obaidi, R.Y., 2004. Palynomorphs and alkane peaks of Campanian Khasib Formation oil used to assess oil source correlation and suggestion for other traps along the migration path, East Baghdad oil field, Iraq, 6th International Conference on Geochemistry, Alexandria University, Egypt, $243-255$.

Al-Beyati, F.M., 1998. Organic geochemical and environmental evaluation study of Chia Gara Formation from selected boreholes in middle Iraq, $\mathrm{PhD}$ thesis, University of Baghdad, Unpublished, $172 \mathrm{pp}$.

Al-Qayim, B., and Saadallah, A., 1992. Petrology of Jurassic Chia Gara Formation, Northern Iraq. J Geol Soc Iraq II 25.

Anaya-Gregorio, A., Armstrong-Altrin, J.S., Machain-Castillo, M.L., Montiel-Garcia, P.C., and Ramos-Vazquez M.A., 2018. Textural and geochemical characteristics of late Pleistocene to Holocene fine-grained deep-sea sediment cores (GM6 and GM7), recovered from the southwestern Gulf of Mexico. J Paleogeo, 7: 1 - 19.

Andersson, P.O.D., Worden, R.H., Hodgson, D.M., Flint, S., 2004. Provenance evolution and chemostratigraphy of a Palaeozoic submarine fan-complex: Tanqua Karoo Basin, South Africa: Marine Petrol Geo, 21: 555 - 577.

Armstrong-Altrin, J.S., and Verma, S.P., 2005. Critical evaluation of six tectonic setting discrimination diagrams using geochemical data of Neogene sediments from known tectonic settings. Sed Geo, 177: 115 - 129.

Armstrong-Altrin, J.S., 2009. Provenance of sands from Cazones, Acapulco, and Bahia Kino beaches, México. Revista Mexican de Ciencias Geologicas, 26: 764 - 782.

Armstrong-Altrin, J.S., Lee, Y.I., Kasper-Zubillaga, J.J., Carranza-Edwards, A., Garcia, D., Eby, N., Balaram, V. and Cruz-Ortiz, N.L., 2012. Geochemistry of beach sands along the western Gulf of Mexico, Mexico: Implication for provenance. Chem Erde Geochem, 72: 345 - 362.

Armstrong-Altrin, J.S., Lee, Y.I., Verma, S.P., and Ramasamy, S., 2004. Geochemistry of sandstones from the Upper Miocene Kudankulam Formation, southern India: Implications for provenance, weathering, and tectonic setting. J Sed Res, 74: $285-297$.

Armstrong-Altrin, J.S., Machain-Castillo, M.L., Rosales-Hoz, L., Carranza-Edwards, A., Sanchez- Cabeza, J. and Ruiz-Fernandez, A.C., 2015. Provenance and depositional history of continental slope sediments in the Southwestern Gulf of Mexico unraveled by geochemical analysis. Continental Shelf Res, 95: 15 - 26.

Armstrong-Altrin, J.S., Nagarajan, R., Madhavaraju, J., Rosales-Hoz, L., Lee, Y.I., Balaram, V., Cruz-Martinez, A. and Avila-Ramirez, G., 2013. Geochemistry of the Jurassic and upper Cretaceous shales from the Molango Region, Hidalgo, eastern Mexico: implications for source-area weathering, provenance, and tectonic setting. Compt Rendus Geosci, 345 (4): 185 - 202.

Armstrong-Altrin, J.S., Verma, S.P., Madhavaraju, J., Lee, Y.I. and Ramasamy, S., 2003. Geochemistry of Late Miocene Kudankulam limestones, South India. Intern Geo Rev, 45: 16- 26.

Asiedu, D.K., Dampare, S., Asamoah-Sakyi, P., Banoeng-Yakubo, B., Osae, S., Nyarko, B.J.B. and Manu, J., 2004. Geochemistry of Paleoproterozoic metasedimentary rocks from the Birim diamondiferous field, southern Ghana: implications for provenance and crustal evolution at the Archean-Proterozoic boundary. Geoch J, 38: $215-228$.

Bakkiaraj, D., Nagendra, R., Nagarajan, R. and Armstrong-Altrin, J.S., 2010. Geochemistry of sandstones from the Upper Cretaceous Sillakkudi Formation, Cauvery Basin, Southern India: Implication for provenance. J Geol Soc India, 76: $453-467$.

Bavinton, O.A. and Taylor, S.R., 1980. Rare earth element abundances in Archean metasediments from Kambalda Western Australia. Geochim Cosmochim Acta, 44: 639 - 648.

Bellen, R.C., Dunnington, H.V., Wetzel, R. and Morton, D., 1959. Lexique Stratigraphique Internal Asie. Iraq. International Geological Congress. Fasc. 10a. Paris, France: Commission on Stratigraphy (in French).

Bhatia, M.R. and Crook, K.A.W., 1986. Trace element characteristics of greywackes and tectonic setting discrimination of sedimentary basins. Contrib Mineral Petrol, 92: 181 - 193.

Bhatia, M.R., 1983. Plate tectonics and geochemical composition of sandstones. J Geol, 91: 611 - 627. 
Bhatia, M.R., 1985. Rare earth element geochemistry of Australian Paleozoic graywackes and mudrocks: provenance and tectonic control. Sed Geo, 45: $97-113$.

Bjorlykke, K., 1974. Geochemical and mineralogical influence of Ordovician island arcs on epicontinental clastic sedimentation: a study of Lower Paleozoic sedimentation in the Oslo Region, Norway. Sedimentology, 21: $251-272$.

Buday, T., 1980. The Regional Geology of Iraq: Stratigraphy and Paleogeography. Vol. I. State Organization for Minerals, Baghdad, p 445, Publications of GEOSURV.

Calvert, S.E., and Pedersen, T.F., 1993. Geochemistry of Recent oxic and anoxic marine sediments: implications for the geological record. Marine Geo, 113 (1-2): $67-88$.

Condie, K.C., 1991. Another look at rare earth elements in shales. Geochim Cosmochim Acta, 55: 2527 - 2531.

Condie, K.C., 1993. Chemical composition and evolution of the upper continental crust: contrasting results from surface samples and shales. Chem Geol, 104: 1 - 37.

Cox, R., Lowe, D.R., and Cullers, R.L., 1995. The influence of sediment recycling and basement composition on evolution of mudrock chemistry in the southwestern United States. Geochim Cosmochim Acta, 59 (14): $2919-2940$

Cullers, R.L. and Podkovyrov, V.N., 2000. Geochemistry of the Mesoproterozoic Lakhanda shales in southeastern Yakutia, Russia: implications for mineralogical and provenance control, and recycling. Precamb Res, 104: $77-93$.

Cullers, R.L., 1988. Mineralogical and Chemical Changes of Soil and Stream Sediment Formed by Intense Weathering of the Danberg Granite, Georgia, USA. Lithos, 21: $301-314$.

Cullers, R.L., 1994. The controls on major and trace element variation of shales, siltstones, and sandstones of Pennsylvanian-Permian age from uplifted continental blocks in Colorado of platform sediment in Kansas, USA. Geochim Cosmochim Acta, 58: 4955 - 4972.

Cullers, R.L., 1995. The controls on the major and trace element evolution of shales, siltstones and sandstones of Ordovician to tertiary age in the Wet Mountain region, Colorado, U.S.A. Chem Geol, 123 (1- 4): 107 - 131.

Cullers, R.L., 2000. The geochemistry of shales, siltstones, and sandstones of Pennsylvanian- Permian age, Colorado, USA: implications for provenance and metamorphic studies. Lithosphere, 51: 181 - 203.

Cullers, R.L., 2002. Implication of elemental concentrations for provenance, redox conditions, and metamorphic studies of shales and limestones near Pueblo, USA. Chem Geo, 191 (4): 305 - 327.

Cullers, R.L., B. Bock, and C. Guidotti. 1997. Elemental distributions and neodymium isotopic compositions of Silurian metasediments, western Maine, USA: Redistribution of the rare earth elements. Geochim Cosmochim Acta, 61 (9): 1847-1861.

Cullers, R.L., Chaudhuri, S., Arnold, B., Lee, M., and Wolf, C.W., 1975. Rare earth distributions in clay minerals and lay-sized fractions of Lower Permian Havensville and Eskridge shales of Kansas and Oklahoma. Geochim Cosmochim Acta, 39: 1691 -1703.

Cullers, R.L., Chaudhuri, S., Kilbane, N., and Koch, R., 1979. Rare earths in size fractions and sedimentary rocks of Pennsylvanian- Permian age from the mid-continent of the USA. Geochim Cosmochim Acta, 43: 1285 1302.

Dai, S., Graham, I.T., and Ward, C.R., 2016. A review of anomalous rare earth elements and yttrium in coal. Int J Coal Geol, 159: $82-95$.

Deepulal, P.M., Kumar, T.R., and Sujatha, C.H., 2012. Behavior of REEs in a tropical estuary and adjacent continental shelf of southwest coast of India: evidence from anomalies. J Earth Sys Sci, 121: 1215 - 1227.

Dhannoun, H.Y., and Al- Dlemi, A.M.S., 2013. The relation between Li, V, $\mathrm{P}_{2} \mathrm{O}_{5}$, and $\mathrm{Al}_{2} \mathrm{O}_{3}$ contents in marls and mudstones as indicators of environment of deposition. Arab J Geosci, 6: $817-823$.

Dill, I.H., 1986. Metallogenesis of early Paleozoic graptolite shales from the Graefenthal Horst (northern BavariaFederal Republic of Germany). Econ Geol, 81: 889 - 903.

Dobrzinski, N., Bahlburg, H., Strauss, H. and Zhang, Q.R., 2004. Geochemical climate proxies applied to the Neoproterozoic glacial succession on the Yangtze Platform, South China. In: G. Jenkins et al. (eds.), The extreme Proterozoic: Geology, Geochemistry and Climate. American Geophysical Union Monograph Series, 146: $13-32$.

Dypvik, H., 1984. Geochemical composition sand depositional conditions of upper Jurassic and lower Cretaceous Yorkshire clays, England. Geol Magaz, 121 (5): 489 - 504.

Fatima, S., and Khan, M.S., 2012. Petrographic and geochemical characteristics of Mesoproterozoic Kumbalgarh clastic rocks, NW Indian Shield: implications for provenance, tectonic setting, and crustal evolution. International Geology Review, 54: 1113 - 1144.

Fedo, C.M., Nesbitt, H.W., and Young, G.M., 1995. Unraveling the effects of potassium metasomatism in sedimentary rocks and paleosols, with implications for paleoweathering conditions and provenance. Geol, 23: $921-924$. 
Floyd, P.A., and Leveridge, B.E., 1987. Tectonic environment of the Devonian Gramscatho basin, south Cornwall: framework mode and geochemical evidence from turbiditic sandstones. J Geo Soci, London, 144: 531 542.

Floyd, P.A., Winchester, J.A., and Park, R.G., 1989. Geochemistry and tectonic setting discrimination using immobile elements. Earth Planet Sci Lett, 27: $211-218$.

Gallala, W., Gaied, M.E., Montacer, M., 2009. Detrital mode, mineralogy and geochemistry of the Sidi Aich Formation (Early Cretaceous) in central and southwestern Tunisia: implications for provenance, tectonic setting and paleoenvironment. J. Afr. Earth Sci., 53: 159 - 170.

Garcia, D., Fonteilles, M., and Moutte, J., 1994. Sedimentary fractionations be- tween Al, Ti, and Zr and the genesis of strongly peraluminous granites. J. Geol, 102: $411-322$.

Gu, X.X., Liu, J.M., and Zheng, M.H., 2002. Provenance and tectonic setting of the Proterozoic turbidities in Hunan, South China: geochemical evidence. J Sed Res, 72: 393 - 407.

Guo, Q.J., Shields, G.A., Liu, C.Q., Strauss, H., Zhu, M.Y., Pi, D.H., Goldberg, T., and Yang, X.L., 2007. Trace element chemostratigraphy of two Ediacaran-Cambrian successions in South China: implications for organo sedimentary metal enrichment and silicification in the early Cambrian. Paleogeog Paleoclimat Paleoecol, 254: $194-216$.

Gupta, S., Banerjee, R., Babu, P.V.R., Parihar, P.S., and Maithani, P.B., 2012. Geochemistry of Uraniferous Banganapalle Sediments in the western part of Palnad Sub-basin, Andhra Pradesh: implications on Provenance and Paleo-weathering. Gondwana Geol. Mag. Sec., 13: $1-14$.

Harnois, L., 1988. The CIW index: a new chemical index of weathering. Sed Geo, 55: $319-322$.

Hatch, J.R., and Leventhal, J.S., 1992. Relationship between inferred redox potential of the depositional environment and geochemistry of the Upper Pennsylvanian (Missourian) Stark shale member of the Dennis Limestone, Wabaunsee County, Kansas, USA: Chem Geo, 99: 65 - 82.

Howarth, M.K., 1992. Tithonian and Berriasian ammonites from the Chia Gara Formation in Northern Iraq. Paleont, 35: $597-655$.

Jassim, S.Z., and Goff, J.C., 2006. Phanerozoic development of the Northern Arabian Plate. In: Jassim SZ, Goff JC, editors. Geology of Iraq. Prague, Czech Republic: Dolin, pp. 15 - 34.

Jones, B., and Manning, D.A.C., 1994. Comparison of geochemical indices used for the interpretation of palaeoredox conditions in ancient mudstones. Chem Geo, 111 (4): 111 - 129.

Joo, Y.J., Lee, Y.I., and Bai, Z., 2005. Provenance of the Qingshuijian Formation (Late Carboniferous), NE China: Implications for tectonic processes in the northern margin of the North China block. Sed Geo, 177: 97 114.

Kimura, H., and Watanabe, Y., 2001. Oceanic anoxia at the Precambrian-Cambrian boundary. Geol, $29: 995$ - 998.

Madhavaraju, J., and González-León, C.M., 2012. Depositional conditions and source of rare earth elements in carbonate strata of the Aptian-Albian Mural Formation, Pitaycachi section, northeastern Sonora, Mexico: Rev Mexi de Cien Geol, 29: 478 - 491.

Madhavaraju, J., and Ramasamy, S., 1999. Rare earth elements in limestones of Kallankurichchi Formation of Ariyalur Group, Tiruchirapalli, Cretaceous, Tamil Nadu. J Geol Soc India, 54: 291 - 301.

Madhavaraju, J., Loser, H., Lee, Y.I., Lozano-Santacruz, R., and Pi-Puig, T., 2016. Geochemistry of Lower Cretaceous limestones of the Alisitos Formation, Baja California, Mexico: Implications for REE source and paleo-redox conditions: J South Amer Earth Sci, 66: 149 - 165.

McKirdy, D.M., and Hall, P.A., 2011. Paleoredox status and thermal alteration of the lower Cambrian (series 2) Emu Bay shale Lagerstätte, South Australia. Australian J Earth Sci, 58: 259 - 272.

McLennan, S., Hemming, S., McDaniel, D., and Hanson, G., 1993. Geochemical approaches to sedimentation, provenance, and tectonics. Geol Soc Am Spec Pap, 284: 21 - 40.

McLennan, S.M., and Taylor, S.R., 1991. Sedimentary rocks and crustal evolution: tectonic setting and secular trends. J Geo, 99: 1 - 21.

McLennan, S.M., 1989. Rare earth elements in sedimentary rocks: influ- ence of provenance and sedimentary processes. Rev Miner Geochem, 21: $169-200$.

McLennan, S.M., 2001. Relationships between the trace element composition of sedimentary rocks and upper continental crust. An Electronic J Earth Sci, 2: 1525 - 2027.

McLennan, S.M., Taylor, S.R., and Kroner, A., 1983. Geochemical evolution of Archean shales from South Africa. The Swaziland and Pongola Supergroups. Precamb Res, 22 (1-2): 93 - 124.

McLennan, S.M., Taylor, S.R., Mcculloch, M.T., and Maynard, J.B., 1990. Geochemical and Nd-Sr isotopic composition of deep-sea turbidities: crustal evolution and plate tectonic associations. Geochim Cosmochim Acta, 54: 2015 - 2050 .

Mohialdeen, I.M.J., 2007. Stratigraphy and sedimentology of organic rich limestones of the Chia Gara Formation, Rania area, Sulaimani, Kurdistan Region, NE Iraq. J Zankoy Sulaimani, 10A (1): 91 - 102. 
Mohialdeen, I.M.J., 2008. Source rock appraisal and oil/source correlation for the Chia Gara Formation, Kurdistan, $\mathrm{N}$ Iraq, $\mathrm{PhD}$ thesis, Unpublished, University of Sulaimani, $140 \mathrm{pp}$.

Mohialdeen, I.M.J. and Al-Beyati, F.M., 2007. Sedimentology and hydrocarbon generation potential of Middle Tithonian-Berriasian Chia Gara Formation, well K-109, Kirkuk Oil Field, NE Iraq. Kirkuk Univ J, 2 (1): $27-43$.

Moosavirad, S.M., Janardhana, M.R., Sethumadhav, M.S., and Narasimha, K.N.P., 2012. Geochemistry of Lower Jurassic sandstones of Shemshak Formation, Kerman Basin, Central Iran: provenance, source weathering and tectonic setting. J Geol Soc India, 79: 483 - 496.

Mortazavi, M., Moussavi-Harami, R., Mahboubi, A. and Nadjafi, M., 2013. Geochemistry of the Late JurassicEarly Cretaceous shales (Shurijeh Formation) in the intracontinental Kopet-Dagh Basin, northeastern Iran: implication for provenance, source weathering, and paleoenvironments. Arab J Geosc, 7 (12): 5353 - 5366.

Nagarajan, R., Armstrong-Altrin, J.S., Nagendra, R., Madhavaraju, J., and Moutte, J., 2007a. Petrography and geochemistry of terrigenous sedimentary rocks in the Neoproterozoic Rabanpalli Formation, Bhima Basin, southern India: Implications for paleoweathering condition, provenance, and source rock composition. J Geol Soc India, 70 (2): $297-312$.

Nagarajan, R., Madhavaraju, J., Nagendra, R., Armstrong-Altrin, J.S. and Moutte, J., 2007b. Geochemistry of Neoproterozoic shales of Rabanpalli Formation, Bhima basin, northern Karnataka, southern India: implications for provenance and paleoredox conditions. Rev Mexic de Cien Geol, 24 (2): 150 - 160.

Nath, B.N., Bau, M., Ramalingeswara Rao, B., and Rao, Ch.M., 1997. Trace and rare earth elemental variation in Arabian Sea sediments through a transect across the oxygen minimum zone: Geochim Cosmochim Acta, 61 (12): $2375-2388$.

Nesbitt, H.W., and Young, G.M., 1982. Early Proterozoic climates and plate motions inferred from the major element chemistry of lutites. Nature, 299: $715-717$.

Nesbitt, H.W., and Young, G.M., 1984. Prediction of some weathering trends of plutonic and volcanic rocks based upon thermodynamic and kinetic consideration. Geochim Cosmochim Acta, 48: 1523 - 1534.

Nesbitt, H.W., Markovic, G. and Price, R.C., 1980. Chemical processes affecting alkalie sand alkaline earths during continental weathering. Geochim Cosmochim Acta, 44: 1659 - 1666.

Odisho, K.Y., and Othman, R.S., 1992. Preliminary geochemical evaluation of hydrocarbon source rocks in northern part of Iraq. Iraqi Geol J, 25: 136 - 153.

Osae, S., Asiedu, D.K., Banoeng-Yakubo, B., Koeberl, C., and Dampare, S.B., 2006. Provenance and tectonic setting of Late Proterozoic Buem sandstones of southeastern Ghana: Evidence from geochemistry and detrital modes. J African Earth Sci, 44: 85 - 96.

Pattan, J.N., Masuzawa, T., Borole, D.V., Parthiban, G., Jauhari, P., and Yamamoto, M., 2005. Biological productivity, terrigenous influence and noncrustal elements supply to the central Indian Ocean Basin: paleoceanography during the past $1 \mathrm{Ma}$. J Earth Sys Sci, 114: $63-74$.

Ramos-Vázquez, M.A., Armstrong-Altrin J.S., Rosales-Hoz L., Machain-Castillo M.L., and Carranza-Edwards A., 2017. Geochemistry of deep-sea sediments in two cores retrieved at the mouth of the Coatzacoalcos River delta, western Gulf of Mexico, Mexico. Arab J Geosc, 10 (6): 1 - 19.

Raza, M., Ahmad, A.H.M., Khan, M.S., and Khan, F., 2012. Geochemistry and detrital modes of Proterozoic sedimentary rocks, Bayana Basin, north Delhi fold belt: implications for provenance and source-area weathering. International Geo Rev, 54 (1): 111-129.

Raza, M., Dayal, A.M., Khan, A., Bhardwaj, V.R., and Rais, S., 2010. Geochemistry of Lower Vindhyan clastic sedimentary rocks of Northwestern Indian shield: implications for composition and weathering history of Proterozoic continental crust. J Asian Earth Sci, 39: 51 - 61.

Rimmer, S.M., 2004. Geochemical paleoredox indicators in Devonian-Mississippian black shales, Central Appalachian Basin (USA). Chem Geo, 206: 373 - 391.

Roaldset, E., 1973. Rare earth elements in Quaternary clays of the Numedal area, southern. Lithos, 6: 349 - 357.

Roser, B.P., Korsch, R.J., 1988. Provenance signatures of sandstone- mudstone suites determined using a discriminate function analysis of major element data. Chem Geo, 67: $119-139$.

Roy, P.D., Caballero, M., Lozano, R., and Smykatz-Kloss, W., 2008. Geochemistry of late Quaternary sediments from Tecocomulco lake, central Mexico: Implication to chemical weathering and provenance. Chem Erde Geochem, 68: 383 - 393.

Sabaou, N., Ait-Salem, H., Zazoun, R.S., 2009. Chemostratigraphy, tectonic setting and provenance of the CambroOrdovician clastic deposits of the subsurface Algerian Sahara. J Africa Earth Sci, 55: 158 - 174.

Saha, S., Banerjee, S., Burley, S.D., Ghosh, A., and Saraswati, P.K., 2010. The influence of flood basaltic source terrains on the efficiency of tectonic setting discrimination diagrams: an example from the Gulf of Khambhat, western India. Sed Geo, 228 (1-2): 1 - 13. 
Salae, A.T., 2001. Stratigraphy and sedimentology of the upper Jurassic succession, NE Iraq, MSc Thesis, Unpublished, University of Baghdad, 95pp.

Selvaraj, K., and Chen, C.T.A., 2006. Moderate chemical weathering of subtropical Taiwan: Constraints from solidphase geochemistry of sediments and sedimentary rocks. J Geo, 114 (1): $101-116$.

Sharland, P.R., Archer, R., Casey, D.M., Davies, R.B., Hall, S.H., Heward, A.P., Horbury, A.D., and Simmons, M.D., 2001. Arabian Plate Sequence Stratigraphy. Geo Arabia, Special Publication 2. Manama, Bahrain: Gulf PetroLink.

Shaw, T.J., Geiskes, J.M. and Jahnke, R.A., 1990. Early diagenesis in differing depositional environments: the response of transition metals in pore water. Geochim Cosmochim Acta, 54 (5): 1233 - 1246.

Spalletti, L.A., Limarino, C.O., and Pinol, F.C., 2012. Petrology and geochemistry of carboniferous siliciclastic from the Argentine frontal cordillera: a test of methods for interpreting provenance and tectonic setting. J S Am Earth Sci, 36: $32-54$.

Taylor, S.R., and McLennan, S.M., 1985. The Continental Crust: Its Composition and Evolution. Blackwell, Oxford, UK, $349 \mathrm{pp}$.

Tobia, F.H., and Mustafa, B.H., 2016. Geochemistry and mineralogy of the Al-rich shale from Baluti Formation, Iraqi Kurdistan region: implications for weathering and provenance. Arab J Geosci, DOI 10.1007/s12517016-2762-6.

Tobia, F.H., and Shangola, S.S., 2016. Mineralogy, geochemistry, and depositional environment of the Beduh shale (Lower Triassic), Northern Thrust Zone, Iraq. Turk J Earth Sci, 25: 367 - 391.

Verma, S.P., and Armstrong-Altrin, J.S., 2013. New multi-dimensional diagrams for tectonic discrimination of siliclastic sediments and their application to Precambrian basins. Chem Geol, 355: $117-133$.

Verma, S.P., and Armstrong-Altrin, J.S., 2016. Geochemical discrimination of siliclastic sediments from active and passive margin settings. Sed Geo, 332: $1-12$.

Verma, S.P., Díaz-González, L., and Armstrong-Altrin, J.S., 2016. Application of a new computer program for tectonic discrimination of Cambrian to Holocene clastic sediments. Earth Sci Inf, 9: $151-165$.

Wedepohl, H.K., 1995. The composition of the continental crust. Geochim Cosmochim Acta, 59: 1217 - 1232.

Wignall, P.B., and Myers, K.J., 1988. Interpreting the benthic oxygen levels in mudrocks, a new approach. Geology, 16: $452-455$.

Wouatong, A.S.L., Yerima, B.P.K., Yongue Fouateu, R., Mvondo Ze, A., and Ekodeck G.E., 2013. The origin of etch pits recorded on residual grain surfaces from kaolinized granitic rocks west region Cameroon. Earth Sci Res, 2 (2): $93-110$.

Wronkiewicz, D.J., and Condie, K.C., 1987. Geochemistry of Archean shales from the Witwatersrand Supergroup, South Africa: source-area weathering and provenance: Geochim Cosmochim Acta, 51: 2401 - 2416.

Wronkiewicz, D.J., and Condie, K.C., 1990. Geochemistry and mineralogy of sediments from the Ventersdorp and Transvaal Supergroups, South Africa: cratonic evolution during the early Proterozoic. Geochim Cosmochim Acta, 54: $343-354$.

Yang, J.H., Jiang, S.Y., Ling, H.F., Feng, H.Z., Chen, Y.Q. and Chen, J.H., 2004. Paleocean-graphic significance of redox-sensitive metals of black shales in the basal lower Cambrian Niutitang Formation in GuizhouProvince, South China. Progress Natur Sci J, 14: 152 - 157.

Yarincik, K.M., Murray, R.W., Lyons, T.W., Peterson, L.C., and Haug, G.H., 2000. Oxygenation history of bottom waters in the Cariaco Basin, Venezuela, over the past 578,000 years: results from redox-sensitive metals (Mo, V, Mn, and Fe). Paleocean, 15: $593-604$.

Zaid, S.M., 2017. Petrography and geochemistry of the Middle Miocene Gebel El Rusas sandstones, Eastern Desert, Egypt: implications for provenance and tectonic setting. J Earth Syst Sci, 126 (7): DOI 10.1007/s12040017-0873-4. 